\title{
Advances in drug delivery via electrospun and electrosprayed nanomaterials
}

\author{
This article was published in the following Dove Press journal: \\ International Journal of Nanomedicine \\ 8 August 2013 \\ Number of times this article has been viewed
}

\author{
Maedeh Zamani' \\ Molamma P Prabhakaran² \\ Seeram Ramakrishna ${ }^{1,2}$ \\ 'Department of Mechanical \\ Engineering, ${ }^{2}$ Center for Nanofibers \\ and Nanotechnology, National \\ University of Singapore, Singapore
}

Correspondence: Molamma P Prabhakaran; Seeram Ramakrishna

Center for Nanofibers and

Nanotechnology, Nanoscience and Nanotechnology Initiative, Faculty of Engineering, National University of Singapore, 2 Engineering

Drive 3, Singapore II 17576

Tel +6565168596

Fax +65 68720830

Email nnimpp@nus.edu.sg;

seeram@nus.edu.sg

\begin{abstract}
Electrohydrodynamic (EHD) techniques refer to procedures that utilize electrostatic forces to fabricate fibers or particles of different shapes with sizes in the nano-range to a few microns through electrically charged fluid jet. Employing different techniques, such as blending, surface modification, and coaxial process, there is a great possibility of incorporating bioactive such molecules as drugs, DNA, and growth factors into the nanostructures fabricated via EHD techniques. By careful selection of materials and processing conditions, desired encapsulation efficiency as well as preserved bioactivity of the therapeutic agents can be achieved. The drug-loaded nanostructures produced can be applied via different routes, such as implantation, injection, and topical or oral administration for a wide range of disease treatment. Taking advantage of the recent developments in EHD techniques like the coaxial process or multilayered structures, individually controlled delivery of multiple drugs is achievable, which is of great demand in cancer therapy and growth-factor delivery. This review summarizes the most recent techniques and postmodification methods to fabricate electrospun nanofibers and electrosprayed particles for drug-delivery applications.
\end{abstract}

Keywords: electrospinning, electrospraying, gene delivery, growth-factor delivery, cancer therapy, wound dressing

\section{Introduction}

A drug-delivery system consists of a formulation or a device that enables introduction of a therapeutic agent in the body and enhances its efficacy and safety by controlling the rate, time, and site of release within the body. ${ }^{1}$ This system is aimed at delivering and retaining a sufficient amount of drug for an adequate period of time, and it is also expected to avoid degradation of non-released drugs within the body. As a result, adverse effects associated with undesired fluctuations in drug concentration or ineffectiveness of damaged drug molecules can be alleviated. During the past few decades, polymeric micro/nanostructures have gained huge interest as drug-delivery systems. Drug delivery using polymeric micro/nanostructures is based on the principle that an increased surface area of the drug carrier enhances the drug-dissolution rate. Various methods have been employed to fabricate micro/nanostructures for drugdelivery purposes.

Electrohydrodynamic (EHD) techniques refer to procedures that utilize electrostatic forces as the driving force to fabricate fibers or particles of different shapes with sizes in the nano-range to a few microns through electrically charged fluid jet. The shape, size, morphology, and composition of micro/nanostructures can be modulated through process parameters and material choice, which can consequently customize 
the drug-release profile. Electrospinning and electrospraying are two modes of EHD techniques utilized for the fabrication of continuous ultrafine fibers and particles, respectively. This review focuses on the advanced applications of EHD techniques for incorporation of therapeutic agents into the polymeric carriers in order to develop novel drug-delivery systems, though this technique can also be employed for fabrication of non-polymeric micro/nanostructures.

\section{Electrospinning and electrospraying for drug delivery Electrospinning}

Electrospinning is the most popular and preferred technique for fabrication of nanofibers, due to its simplicity, costeffectiveness, flexibility, potential to scale up, and ability to spin a broad range of polymers. For electrospinning, a strong electrical potential is applied to the polymer liquid (solution or melt), and as a result the electrical charges accumulate on the surface of the liquid droplet at the tip of the capillary. At a critical voltage, the Coulombic repulsion of the charges overcomes the surface tension of the polymer droplet, and a charged jet is ejected from the tip of the droplet. The jet travels towards a grounded electrode, while the solvent gets evaporated, and the resultant fibers are collected on a grounded target. Because of the remarkable characteristics of the nanofibers, such as the high surface area-to-volume ratio, ${ }^{2}$ high interconnected porosity with tunable pore size, ${ }^{3}$ possibilities for efficient surface functionalization, ${ }^{4}$ adjustable surface morphology, ${ }^{5}$ and structural similarity to the extracellular matrix (ECM), they are widely used in tissue regeneration and drug-delivery applications. Various polymers have been electrospun, ranging from natural polymers like collagen, gelatin, chitosan, silk fibroin, and hyaluronic acid to synthetic polymers such as poly(lactic acid) (PLA), poly( $\varepsilon$-caprolactone) (PCL), polyethylene oxide, and copolymers, such as poly (L-lactide-co-caprolactone) and poly(lactic-co-glycolic acid) (PLGA), to fabricate scaffolds with desired properties for specific tissue regeneration and drug-delivery applications. ${ }^{6}$ Drug-loaded nanofibrous membrane can be applied topically for skin and wound healing ${ }^{7}$ or as a postoperation implant for antibiotic, antifungal, antimicrobial, ${ }^{8}$ and anticancer ${ }^{9}$ drug delivery.

Electrospinning provides the opportunity for direct encapsulation of drugs into the electrospun fibers. Both hydrophobic (eg, rifampin, paclitaxel) and hydrophilic drugs (eg, tetracycline hydrochloride, doxorubicin hydrochloride) and biomacromolecules, such as proteins and DNA, can be encapsulated to the electrospun fibers. The high surface area of nanofibers as well as three-dimensional open porous structure help to reduce the constraint towards drug diffusion, resulting in a more efficient drug-release system. ${ }^{10}$ Moreover, local drug delivery using electrospun fibers can decrease the minimum required dosage of the drug, leading to a less systemic absorption, and reduce unwanted side effects. Compared to other forms of drug carriers, such as liposomes, hydrogels, and nano/microspheres produced by conventional methods, electrospun nanofibers are able to improve significantly drug-encapsulation efficiency and reduce the burst release via proper selection of a drug-polymer-solvent system or electrospinning technique. ${ }^{11}$ Also, electrospun fibrous mats would facilitate the diffusion of drug molecules into the surrounding medium in comparison to conventional films produced by a solution-casting technique. ${ }^{12}$ Due to the high porosity of electrospun membranes, the by-products produced by degradation of the polymeric materials will not accumulate at the site of implantation. Moreover, electrospun membranes can be cut to any size and shape, making them suitable for target clinical application. ${ }^{13}$

\section{Electrospraying}

Electrospraying, also called EHD atomization (EHDA), a modified version of the electrospinning process, is a promising technique for preparation of micro- and nanoparticles suitable as drug-delivery systems. Polymeric micro- and nanoparticles are capable of being administered as oral, injectable, inhalable, topical, and local drug-delivery systems. Solvent evaporation, ${ }^{14}$ single and double emulsion, ${ }^{15-18}$ spray-drying, ${ }^{19}$ porous glass membrane emulsification, ${ }^{20}$ and coacervation ${ }^{21}$ are the commonly employed techniques for fabrication of biodegradable polymeric micro- and nanoparticles. However, most of these methods have some disadvantages, such as low drug-loading efficiency, ${ }^{22}$ limitations to scale up, particle-size polydispersity, low ability to fabricate small particles (below $100 \mathrm{~nm}$ ), and difficulties for incorporation of hydrophilic drugs. ${ }^{15,23,24}$ Moreover, the need for particle separation from dispersing solution or even nondegradable surfactants employed in some of the fabrication processes might cause additional difficulties. ${ }^{25}$ At the same time, the inactivation or degradation of drug is possible, due to organic solvent exposure, high shear stress, high temperature, and aqueous organic interfaces, especially for the biomacromolecules. ${ }^{18,26,27}$

Using the technique of electrospraying, the drawbacks associated with conventional particle-producing methods might be overcome. Similar to the electrospinning process, fabrication of drug-loaded polymeric particles via electrospraying can be performed using a solution of polymer 
and drug in a sufficiently conductive solvent. The principle of electrospraying is similar to the electrospinning process, and by altering the solution properties, eg, concentration, as well as processing parameters such as flow rate and applied voltage, a continuous and charged jet can be broken down into droplets, resulting in particles of different size and shape. ${ }^{28,29}$ Figure 1 schematically shows how solution/ processing parameters can effectively control the resultant micro/nanostructure morphology, leading to either electrospinning or electrospraying.

The main advantages of EHDA over other conventional encapsulating methods are the capability of obtaining a higher loading efficiency, narrow particle-size distribution, and ease of particle synthesis due to single-step processing. ${ }^{30}$ Furthermore, electrospraying would eliminate the need for using a surfactant or additional template. ${ }^{22}$

Size polydispersity of particles is a constraint limiting the potential application of micro/nanoparticles, eg, in the inhalable drug-delivery system, where the aerosol particles with homogeneous size and suitable aerodynamic diameter (1-5 $\mu \mathrm{m})$ are essential. ${ }^{25}$ Besides, the dissolution rate of a drug is dependent on the size of both drug and its carrier. Thus, drug absorption/bioavailability in the body could be properly regulated by particle-size modulation, ${ }^{31}$ while the particle cellular uptake is influenced by the size of the polymeric particles. ${ }^{32}$ By selecting a suitable solvent and altering the operating parameters of the primary electrospraying setup, monodispersed particles with very small standard deviation in particle sizes can be obtained. ${ }^{33}$

The typical electrospraying setup consists of three main components: high-voltage power supply, spinneret, and conductive plate as collector. Studies demonstrated that placing a ring connected to the intermediate voltage below the spinneret stabilizes the electrospraying jet, as highly charged particles are strongly attracted by grounded or oppositely charged objects. As a result, more uniform particle size can be achieved. ${ }^{34,35}$ Another possible way to achieve monodisperse particles is to separate the particles according to their mass/ surface-charge density. Hong et al employed a steel plate with a circular hole as ground electrode between the nozzle and the collector to filter satellite particles, allowing the deposition of monodisperse primary particles on the collector. This process was possible because of the differences in run tracks between the two kinds of particles, forming a spray region with an inner core of primary droplets and an outer layer of satellite droplets under the capillary. ${ }^{25}$ However, the efficiency of the process (fabricated particles per hour) will be reduced, as a significant portion of the electrosprayed particles are filtered out using this technique.

Moreover, if solid particles are required for the target application, the droplets should be discharged to prevent Rayleigh disintegration of the sprayed droplets, facilitating par-

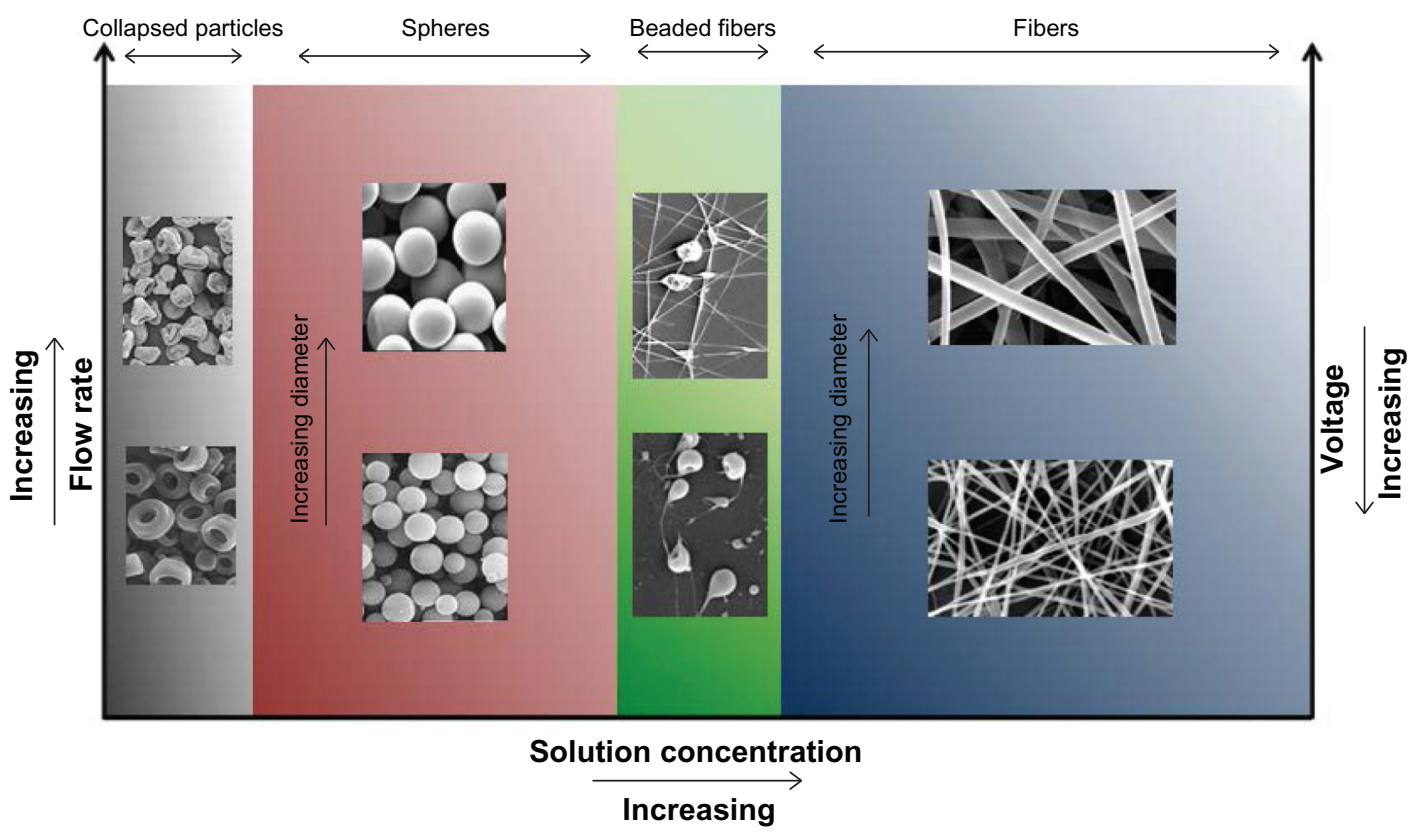

Figure I Schematic illustration of the effect of the concentration, flow rate, and voltage on electrosprayed/electrospun nano/microstructures.

Notes: At very low concentrations, fabricated particles collapse into rings, discs, etc, by varying the electrospraying condition. By increasing the concentration, spherical particles can be formed with different sizes by changing the flow rate and applied voltage. Further increase in concentration results in formation of particle-tail structures or beaded fibers depending on the processing condition. When a critical concentration is reached, uniform fibers are produced. Although these general principles are applicable for most electrospinning/electrospraying conditions, solutions with specific properties such as high conductivity may behave differently. 
ticle handling. A corona needle connected to opposite charges was also tested under the spraying nozzle to neutralize droplets with high electric charges. ${ }^{33-35}$ Control of solvent-evaporation rate is another approach for manipulating particle morphology. During electrospraying, quick solvent evaporation would lead to the formation of porous, wrinkled, or shell-like particles. Although these morphologies are beneficial for special applications, such as pulmonary drug delivery, because of low mass density, uniform spherical drug-loaded particles are more desirable for most applications. A chamber with air or nitrogen flow enclosing the nozzle was described to effectively control the solvent-evaporation rate and particle morphology. ${ }^{34-36}$ Although single-step synthesis of solid particles is considered one of the main advantages of EHDA, a grounded conductive solution can be used as the collector, in case colloidal solutions of particles are required for a particular application. 27,31,37 This method enables integration of probable post electrospraying surface-modification process with a particle-collecting step. ${ }^{30,38-40}$

Considering the specific requirements of suitable drugdelivery devices for different diseases and also the wide variety of drug characteristics, such as stability and solubility, various techniques might be needed to desirably incorporate the target therapeutic agent into electrospun nanofibers or electrosprayed particles. The most popular methods of drugincorporation techniques are discussed here.

\section{Drug-incorporation techniques Blending Electrospinning}

Among all the developed methods for drug loading into nanofibers, blending of the therapeutic agent with polymeric solutions remains the most predominant. For this purpose, the drug is dissolved or dispersed in the polymer solution to achieve encapsulated drug through a one-phase electrospinning method. Although this technique is simple compared to other encapsulation methods, such as emulsion electrospinning or the most complex electrospinning techniques like coaxial electrospinning, some requirements should be met in order to gain the desired results. For example, drug-release behavior is highly dependent on the distribution of drug molecules into electrospun fibers, as well as the morphology of the fibers. In order to attain successful encapsulation of drug into the electrospun nanofibers, the physicochemical properties of polymers as well as their interaction with drug molecules must be precisely considered, as they significantly affect drug-encapsulation efficiency, drug distribution inside the fibers, and kinetics of drug release. In other words, the hydrophobic-hydrophilic properties of drug and polymer should be matched. That is, lipophilic drugs such as rifampicin and paclitaxel and hydrophilic drugs like doxorubicin hydrochloride should be loaded into lipophilic and hydrophilic polymers, respectively, in order to achieve perfect encapsulation of the drug inside the electrospun nanofibers. Lack of solubility of the drug in the polymer solution causes just a dispersion of the drug inside the solution. During electrospinning of such drug dispersions, the drug molecules might migrate on or near the fiber surfaces, resulting in burst release. ${ }^{11}$ In order to achieve sustained release, blending of hydrophilic-hydrophobic polymers using different polymer combinations has been carried out by many researchers, ${ }^{41-44}$ and the results showed that addition of hydrophilic polymers such as gelatin, ${ }^{41}$ polyethylene glycol (PEG), ${ }^{43}$ polyvinyl alcohol (PVA), ${ }^{44}$ or amphiphilic copolymers like PEG-bPLA diblock copolymer ${ }^{42}$ could significantly enhance drugloading efficiency and subsequently reduce the burst release of drugs. For high-molecular-weight therapeutic agents, the drug-release rate was enhanced using hydrophilic polymeric nanofibers. The positive interaction of hydrophilic drug and polymer might limit the tendency of the drug molecules to migrate to the surface of the nanofibers. ${ }^{45}$ Alternatively, copolymerization $^{46,47}$ and polymer modification ${ }^{48}$ have also been studied to enhance the hydrophilic properties of polymeric carriers.

\section{Electrospraying}

With the similarities in the fundamental processes of electrospinning and electrospraying, most of the previously mentioned considerations and limitations of electrospinning can be extended to electrospraying. Similar to electrospinning, blending of the therapeutic agent in a polymer solution is the simplest way for drug encapsulation into the electrosprayed particles. This technique provides the possibility of directly incorporating a drug into a polymeric carrier in a single step, in contrast to methods that require two or more steps to produce the final drug-loaded particles. ${ }^{49}$ Adequate physical interactions between the polymer and the drug are vital for getting sustained and prolonged drug-release behavior. ${ }^{50}$ EHDA is not only a promising technique for nanofabrication but also can be considered an amorphization technique. Amorphization together with nanosize effects are defining mechanisms for enhanced dissolution of poorly water-soluble drugs after encapsulation into the polymeric nanoparticles. ${ }^{51}$ It was also demonstrated that electrospraying is a safe technique for processing several types of cells, ${ }^{52-54}$ and studies have also shown that the activity of proteins could be preserved during this process. ${ }^{55,56}$ 


\section{Surface modification}

\section{Electrospinning}

Surface modification of nanofibers with target biomolecules is another promising method for introducing biofunctionality into them. Using this technique, the therapeutic agent is bound or conjugated to the fiber surfaces, making it structurally and biochemically similar to the native tissue. In such a way, the release of the therapeutics would be attenuated, and the functionality of the surface-immobilized biomolecules could be preserved.$^{57}$ This strategy is usually applied to solve the problem of initial burst release as well as short release time ${ }^{58}$ However, when the target biomolecule is chemically immobilized onto the surface, it would hardly be released. So this technique would be more applicable for gene or growth-factor delivery, where a slow and prolonged release of the therapeutic agent is required. ${ }^{57,59-63}$ For biomolecules like DNA, growth factors (GFs), or enzymes that lose bioactivity and degrade within a few days, conjugating the biomolecules to the fiber surfaces and slowly releasing them into the nearby tissue would significantly preserve their functionality.

However, drugs that are required to be endocytosed or that require interaction with the cell nucleus cannot be immobilized in this way. In such cases, the release rate of immobilized target molecules could be precisely controlled via introduction of responsive materials to local external cues. ${ }^{59}$ In addition to immobilization of drug molecules, surface modification of nanofibers with various chemical compounds can be applied to modulate drug-release profiles from drug-blended nanofibers. For example, fluorination of the nanofiber surfaces caused controlled drug-release rate by introducing hydrophobic functional groups onto the surface of fibers. ${ }^{58}$

\section{Electrospraying}

In contrast to electrospun nanofibers, postelectrospray surface modification of particles is not employed as an approach for surface immobilization of drugs. Instead, it is carried out to modulate the surface properties of the particles to obtain desired results. Since an EHDA device offers the possibility of using a liquid-phase particle-collecting medium, it would be beneficial for stabilization of electrosprayed particle suspension,,$^{31,37}$ and for further particle-surface modifications, such as coating ${ }^{38,39}$ and cross-linking. ${ }^{30,40,64}$ Almería et al reported the application of PVA in deionized water as the collecting medium whereby, PVA uniformly coated the PLGA drug-containing particles by binding onto the PLGA surface ${ }^{38}$ Electrospraying was also applied for preparation of cross-linked doxycycline-loaded chitosan (CS) nanoparticles using tripolyphosphate, a biocompatible cross-linking agent. Aqueous solution of tripolyphosphate was employed as both cross-linking and collecting solution to improve the mechanical and transport properties..$^{30}$ Although considerable attempts have been made to combine particle fabrication and surface modification by electrospraying, there is still great potential for further development for immobilization of functional groups on the surface to facilitate drug delivery, cell uptake, and impart particle-tissue interactions.

\section{Coaxial process}

\section{Coaxial electrospinning}

This is a modified version of electrospinning that enables production of fibers with core-shell morphology. Incorporation of biomolecules such as DNA by blending with polymer solutions caused localization of DNA molecules on the surface of fibers, rather than being encapsulated within the fibers. The transfection efficiency of DNA released from the electrospun fibers was significantly lower than a commercial lipid-based transfection mediation agent, namely Fugene $6 .{ }^{65}$ To improve the functionality of biomolecules, coaxial electrospinning was performed, where the biomolecule solution formed the inner jet and it was co-electrospun with a polymer solution that formed the outer jet. ${ }^{66}$ The shell polymer not only contributes to the sustained and prolonged release of the therapeutic agent, but also plays an essential role in protecting the core ingredient from direct exposure to the biological environment. ${ }^{67}$ The main advantage of this method over the commonly used electrospinning device is that the fibers are fabricated from two separate solutions, minimizing the interaction between aqueous-based biological molecules and the organic solvents in which the polymer is mainly dissolved. As a result, not only the bioactivity of the unstable biological molecules can be preserved but also additional modification processes like lyophilization of plasmid DNA (pDNA) can be avoided. ${ }^{67}$

Successful encapsulation of drug to the core of the coelectrospun fibers is determined by various parameters, eg, shell polymer concentration, core polymer concentration, and molecular weight and drug concentration. Moreover the relative flow rate of the core and shell solutions is a key parameter that determines the encapsulation efficiency of a drug. ${ }^{66}$ Fiber morphology is sometimes critical, and by incorporating low-molecular-weight (3400) PEG as a porogen into the shell of the fibers, accelerated transport of core molecules into the environment was achieved. ${ }^{66}$ In addition to the coreincorporated bioactive molecules, the shell can be contained with bioactive molecules like nonviral gene-delivery vectors, 
which can act as a nonviral delivery vector for encapsulated DNA upon release. ${ }^{67}$

The coaxially electrospun fibers introduced a new generation of tissue-engineering scaffolds that can achieve sustained, local, and efficient gene and growth-factor delivery to cells seeded on the scaffold.$^{56,66,67}$ It was also shown that the GFs released from these coaxial fibers maintained the same level of bioactivity as fresh GFs ${ }^{56}$ At the same time, coaxial electrospinning is capable of reducing virus dissemination and its corresponding inflammatory cytokine secretion, as well as limiting the effect of viral transduction at the site of action. ${ }^{66}$ Although coaxial electrospun nanofibers gained huge interest as gene- and GF-delivery systems, other types of pharmaceutical compounds, such as antibiotic or antioxidant drugs, were also embedded into coaxially electrospun nanofibers for different applications. ${ }^{68-70}$

\section{Coaxial electrospraying}

Electrosprayed drug-blended particles also encounter problems of initial burst release, especially for hydrophilic drugs, and coaxial multicapillary electrospraying was a rescue. By employing this setup, monodisperse core-shell particles with core-embedded drugs were fabricated. Encapsulation of unstable bioactive molecules, such as proteins, enzymes, and antibiotics, without losing their functionality was possible by this method. ${ }^{27}$ Additionally, coaxial electrospinning might help to overcome the problem of particle aggregation by performing polymeric shell modification without altering the drug-contained core solution. ${ }^{51}$ By making a careful selection, it was also possible for the polymeric shell to act as a physical support for core-embedded drug. ${ }^{71}$ However, the size and internal structure of core-shell particles have to be precisely controlled, since the release kinetics is mainly determined by drug diffusion through the polymeric shell. ${ }^{37}$ It was demonstrated that the coaxial dual-capillary electrospray is capable of producing monodisperse drug-loaded particles with high encapsulation efficiency. ${ }^{37}$

Coaxial tricapillary electrospraying is another technique for fabrication of particles with core-middle-shell structure, which can be used for the production of multidrug-loaded particles. Recent clinical studies have demonstrated higher effectiveness of multiple drug delivery compared to single drug administration, eg, in cancer therapy. ${ }^{72,73}$ However, blended or bilayered particles loaded with multiple drugs do not allow control of individual drug release. ${ }^{74}$ Several techniques, such as microfluidic techniques and layerby-layer self-assembly and emulsion, were suggested to enable separate regulation of multiple drug-release kinetics.
However, each of these methods suffers from some difficulties. For example, multiple-emulsion procedures are essential for incorporation of drugs with different hygroscopic properties, leading to low encapsulation efficiency and probably drug inactivation. Layer-by-layer self-assembly is also limited, due to multiple-step procedures utilized for sequential adsorption of oppositely charged polyelectrolytes to produce multilayered particles. ${ }^{37}$ Using coaxial tricapillary electrospraying, various drugs can easily be incorporated into core, middle, and outer layers of particles, separately, producing multidrug-loaded particles in one step, and this technique can also offer individual release of multiple drugs from the same device. In this way, not only can the aforementioned advantages for dual-capillary electrospray be achieved but also the need for frequent drug administration can be reduced, resolving problems associated with complex prescriptions. $^{74}$

\section{Emulsion electrospinning}

The main drawback of coaxial electrospinning is the complexity in the design and EHD behavior of the process, whereby the spinning parameters, interfacial tension, and viscoelasticity of the two polymers must be precisely controlled. Even after doing so, only a portion of the produced fibers form the core-shell structure. ${ }^{75}$ Compared to electrospinning of polymer solution-containing drug dispersions, the hydrophilic biomolecule is expected to be encapsulated inside the fibers instead of escaping onto the fiber surfaces using emulsion electrospinning. ${ }^{76}$ In emulsion electrospinning, the drug or aqueous protein solution is emulsified within a polymer solution, which is referred to as the oil phase, and after electrospinning, the biomolecule-loaded phase can be distributed within the fibers if a low-molecular-weight drug is used $^{77}$ or form a core-shell fibrous structure when macromolecules are incorporated in the aqueous phase. ${ }^{75,76,78,79}$ The ratio of aqueous/polymer solution is one of the effective parameters that affects the distribution of the biomolecules within the fibers, a phenomenon that can subsequently affect the release profiles, structural stability, and bioactivity of the encapsulated proteins. ${ }^{75}$

The potential advantage of emulsion electrospinning over the conventional blending technique is that the drug and polymer are dissolved in appropriate solvents, eliminating the need for a common solvent. As a result, various hydrophilic drugs and hydrophobic polymeric combinations can be applied, and during this process the drug contact with organic solvent is minimal. ${ }^{77}$ However, compared to coaxial electrospinning, emulsion electrospinning would still 
cause damage or degradation of unstable macromolecules like pDNA, probably because of the shearing force or the interface tension between the aqueous and organic phases of the emulsion. Hence, further modifications like condensation of pDNA might be useful to protect pDNA from denaturation. ${ }^{76,78}$ Another disadvantage of emulsion electrospinning is that the emulsification and ultrasonication procedures would increase the (protein) core contact with the solvent by disruption of the aqueous protein droplets, thereby increasing the probability of protein damage. ${ }^{75}$

Though extremely hydrophobic polymers can be used in emulsion electrospinning, the affinity or compatibility between drug and polymer might also influence the distribution of drugs within the fibers. It was demonstrated that copolymerization of hydrophobic polymers with hydrophilic polymers such as PEG would positively affect drug distribution. ${ }^{77}$

\section{Other approaches in electrospinning}

Researchers are always trying to develop or modify existing techniques to overcome limitations and subsequently improve the properties of drug-delivery systems. One of the recent advances in developing more efficient drug-delivery systems has been to include combination therapies, ie, administration of multiple drugs with the same or different therapeutic effects aiming to improve the treatment results. The most important factor in multidrug-delivery systems is independent controlled release of drugs, particularly in cancer or other complex diseases, so as to prevent multidrug resistance. However, independent controlled release of each drug in a multidrug-blended carrier cannot be easily obtained. Incorporation of different drugs into the same carrier provides the same diffusion pathway and matrix-degradation rate, and hence the individual drug-release rate cannot be optimized. ${ }^{80}$ To realize a time-programmed multidrugdelivery system with a single formulation, a drug carrier that enables presetting of the release profile of all component drugs is required. In this context, sequential electrospinning was developed to construct multilayered electrospun mats consisting of various drugs and basement membranes. In this system, the fiber size and each layer thickness were effective variables in controlling the drug-release rate and timing. A time-programmed, retarded drug release was obtained with potential application for sequential chemotherapy using multiple anticancer drugs. ${ }^{81}$ Incorporation of drug-contained polymeric particles into electrospun fibers was also studied for multiple drug-delivery applications. ${ }^{80,82}$ Naproxenand rhodamine B-loaded CS particles were dissolved in PCL solution, forming a chain of nanoparticles at the core of the ultrafine fibers after electrospinning. ${ }^{80}$

Multilayer-coated nanofibers are another innovative approach for producing nanofibrous drug-delivery systems. This method combines the unique properties of electrospun nanofibers, such as high surface area, with proven advantages of polyelectrolyte multilayer structures for drug-delivery applications. Polyelectrolyte multilayer structures can be produced through layer-by-layer adsorption of polymers, which can form electrostatic or hydrogen bonding or even acid-base pairing. ${ }^{83}$ The feasibility of producing multilayercoated fibers for drug-delivery purposes was demonstrated by Chunder et al, where nanofibers of two oppositely charged weak polyelectrolytes, polyacrylic acid (PAA) and allylamine hydrochloride $(\mathrm{PAH})$ containing methylene blue as a cationic model drug were fabricated using electrospinning. Using $\mathrm{PAA} / \mathrm{PAH}$ as the polymer matrix, it was possible to control the drug-release rate by adjusting the $\mathrm{pH}$. Further control over methylene blue release was achieved by employing the deposition of a hydrophobic layer of perfluorosilane onto the drug-loaded nanofibers. Surface coating was also performed using PAA/poly( $N$-isopropylacrylamide), acquiring thermosensitive drug-release properties. ${ }^{83}$ In another attempt, alginate and CS were used to coat PVA electrospun fibers, aiming to orally deliver preserved Lactobacillus to the large intestine. CS and alginate get removed in the stomach and small intestine, due to their solubility in acid and neutral conditions, respectively, leaving behind Lactobacillusloaded PVA fibers, and the release of Lactobacillus into the large intestine was possible due to its swelling properties. Figure 2 shows the suggested mechanism of drug release of the prepared multilayer-coated nanofibers. ${ }^{84}$ In addition to these investigated modifications of nanofiber processing, modulations in the electrospinning setup were also tested by researchers. Two-stream electrospinning was employed to make composite nanofibrous meshes from different polymers/drugs. In this way, two separate polymers/drugs with required functionalities can be electrospun simultaneously to achieve combined properties by independent fiber populations. ${ }^{85}$

\section{Applications Electrospun nanofibers as drug-delivery systems}

To date, drug-incorporated electrospun nanofibers have been used for treatment of different diseases, attaining various levels of success. The membranes were mainly applied as topical or implanted drug-delivery systems, though reports on the oral 


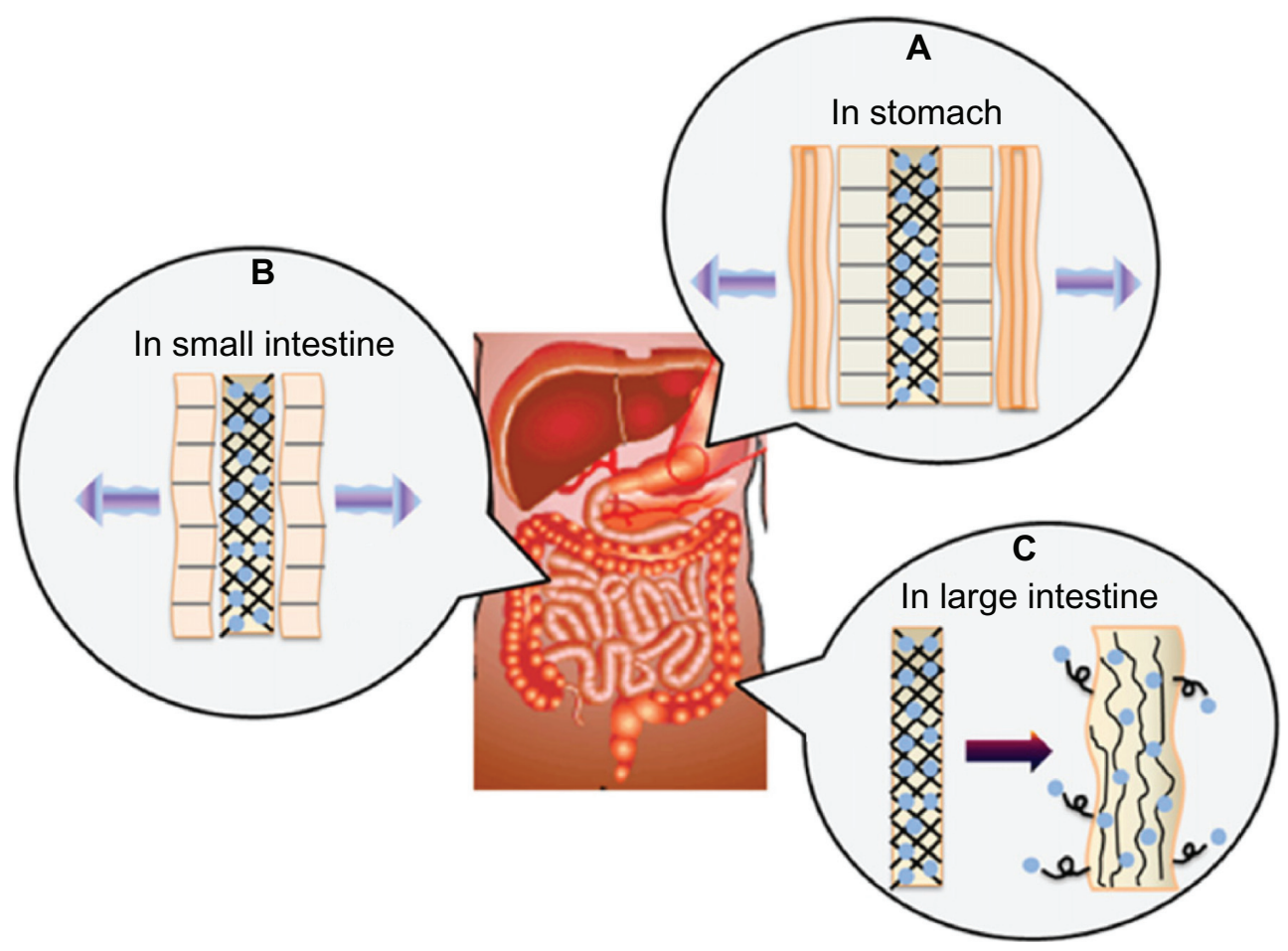

Figure 2 (A-C) Drug-release mechanism suggested for chitosan/alginate/polyvinyl alcohol multilayered nanofibrous drug-delivery system.

Reprinted from J Ind Eng Chem. Im JS, Lee SK, Bai BC, Lee YS. Prediction and characterization of drug release in a multi-drug release system. I8(I):325-330. Copyright (20I2) with permission from Elsevier. ${ }^{84}$

administration of electrospun nanofibers are also available. The most frequent applications of nanofibrous drug-delivery systems are discussed in the following sections.

\section{Transdermal systems and wound dressings}

Wound dressings assist in protecting the wound from exogenous microorganisms, absorbing exudate and finally enhancing cosmetic appearance. ${ }^{86} \mathrm{An}$ ideal wound dressing not only preserves the wound from infections but also accelerates the wound-healing process. ${ }^{87}$ However, inert wound dressings are not completely successful in preventing infections wherein the wound-healing process is prolonged, particularly for burns, split-skin graft-donor sites, pressure sores, and diabetic ulcers. As a result, bioactive dressings containing antibiotics are usually required in the initial period of wound healing. ${ }^{44}$ To date, bioactive wound dressings have been developed with such structures as films, hydrogels, sponges, and foams ${ }^{44}$ Recently, electrospun nanofibers showed great capability for wound dressing due to their special characteristics, such as extremely high surface area, and thus electrospun nanofibers can efficiently absorb exudates, adjusting the wound moisture. ${ }^{44,86}$ Although high porosity of nanofibrous membrane effectively contributes to air permeability, providing required oxygen for cell respiration, the relatively small pore size can preserve the wound from bacterial infections. Enhanced hemostasis and more flexibility in dressing, which is required for entirely covering complicated wounds, as well as great possibilities for functionalization with various bioactive molecules and mechanical strength are achievable benefits through nanofibrous dressings. Finally, from the aesthetic point of view, nanofibers offer the superior advantage of scar-free regeneration by conducting the normal skin-cell proliferation. ${ }^{88,89}$ Electrospun nanofibers of PVA, poly(vinyl acetate), and a blend loaded with ciprofloxacin hydrochloride were studied as wound dressings, and it was demonstrated that the addition of poly(vinyl acetate) to PVA nanofibers decreased the drug-release rate at earlier stages, prolonging drug release ${ }^{44}$ In another study, the dynamic interactions of fusidic acid-loaded electrospun PLGA nanofibers with wound bacteria were explored. In vitro microbiological tests showed bacterial colonization of fibers forming a thick layer of biofilm. Interestingly, it was observed that the preexposure of membrane to wound bacteria caused a significant improvement in drug-release rate, which was the consequence of the changes in fiber morphology as well as the reduction in $\mathrm{pH}$ of the incubation medium. However, loading of adequate concentrations of fusidic acid into the nanofibers remarkably prohibited bacterial biofilm formation. ${ }^{90}$ The ability of epidermal GF (EGF)-loaded silk nanofibers was 
also demonstrated to contribute strongly towards the healing process by decreasing the time of wound closure. ${ }^{91}$

A transdermal drug-delivery system (TDDS) is designed to support and facilitate the delivery of target agents into the body through the skin. ${ }^{92}$ In addition to wound-dressing applications, electrospun nanofibers can also be used as a TDDS. It is an alternative way of oral administration of sensitive drugs, particularly the ones that are greatly affected by first-pass metabolism..$^{93}$ Moreover, controlled drug release from a TDDS significantly improves drug efficiency, because of reduced fluctuations in the therapeutic agent level in the body. However, a limited number of drugs can effectively pass through skin because of the low drug permeability of skin, and hence drug administration is often limited to therapeutic agents with low molecular weight (up to a few hundred Daltons), high hydrophobic properties, and low concentrations (up to milligrams per day or less). Therefore, the transdermal delivery of large hydrophilic drugs remained problematic. ${ }^{92,94}$

Electrospun nanofibers for transdermal delivery of vitamins and anti-inflammatory and antioxidant drugs employing hydrogel polymers like PVA and cellulose acetate as drug carriers were successfully explored. ${ }^{2,12,92,95-98}$ Yun et al fabricated a TDDS by electrospinning of poly(vinyl alcohol)/ poly(acrylic acid)/multi-walled carbon nanotube (PVA/PAA/ MWCNT) nanofibers containing ketoprofen, and the swelling properties as well as drug-release behavior of the nanofibers was significantly altered by modulating the concentration of MWCNTs, oxyfluorination conditions used for introducing hydrophilic functional groups on MWCNTs, and applied electric voltage. Cell-viability tests confirmed more than $80 \%$ cell viability, which is an acceptable rate for a biocompatible material. ${ }^{92}$ Comparisons between PVA nanofibrous membrane and PVA cast film showed enhanced swelling, weight loss, and drug-release rate for the former structure. Transdermal diffusion through pigskin experiments showed that the drug molecular weight has a more significant effect on determining drug-release characteristics than the hygroscopic properties of the drug. ${ }^{12}$ Herbal pharmaceutical compounds were also successfully incorporated in electrospun nanofibers in order to develop topical/transdermal or wound-dressing devices. Ultrafine cellulose acetate electrospun fibers containing asiaticoside, a herbal extract, either as a pure agent or a crude extract were fabricated. Drug-release results in immersion mode were in agreement with previous conclusions about faster drug release from nanofibrous mats compared to drug-loaded films. However, much slower drug release was obtained using transdermal diffusion through a pigskin method compared to the total immersion technique. ${ }^{2}$

\section{Cancer therapy}

Cancer therapy is still a point of challenge in the world of medical science, and in spite of admirable achievements, a considerable number of deaths occur each year because of the ineffectiveness of current therapeutic strategies to treat cancer. To date, a wide range of cancer therapeutic agents and techniques have been developed to effectively treat various cancers via mechanisms such as metabolism revision, interaction with microtubule degradation to improve mitotic arrest, reduction of cell motility, and interruption to intercellular signal transmission. ${ }^{6,99}$ However, systemic administration (both orally and intravenously) of anticancer drugs is associated with some drawbacks, such as poor solubility and instability of the drug in the biological environment, low concentration of the drug around the tumor site, low efficacy for solid tumors, undesired side effects on healthy tissues such as neutropenia or cardiomyopathy, and high rate of elimination by the reticuloendothelial system. ${ }^{13,100}$

Formulations based on liposomes, micelles, hydrogels, and nanoparticles were explored to deal with these problems, leading to more efficient, safe, viable, sustained, and eventargeted drug-delivery systems for cancer chemotherapy. But the common polymeric drug-delivery systems like nano- or microspheres, liposomes, and hydrogels often show sharp burst drug release and sometimes low drug-loading efficiency because of the hydrophobic nature of the drug. ${ }^{11,13}$ In the case of solid tumors, a combination of surgical operation for removing the tumor with subsequent chemotherapy or radiation therapy is the normal procedure to reduce the probability of recurrence. In order to achieve a prolonged period of function, maximized efficiency, and minimized unwanted side effects to healthy tissues, methods such as local and sustained postsurgical drug delivery have been explored. ${ }^{101}$ Electrospun fabrics loaded with anticancer drugs can be conveniently inserted to cover the solid tumor site, and this not only provides high local dosage with incorporation of small amounts of the drug but also reduces the need for frequent administrations, resulting in patient convenience.

The early study by Zeng et al involved the encapsulation of the lipophilic drug paclitaxel, the hydrophilic drug doxorubicin hydrochloride, and lipophilic doxorubicin base in electrospun poly(L-lactic acid) (PLLA) nanofibers, aiming to explain when and why the burst release happens from electrospun fibers. Desirable encapsulation of paclitaxel and doxorubicin base was found due to acceptable polymer-drug-solvent compatibility, but doxorubicin hydrochloride was observed near the surfaces of PLLA fibers causing an obvious burst release. ${ }^{11}$ However, the inhibitory activity against cancer cells 
was not assessed by these researchers. Recently, the inhibitory effect of released dichloroacetate from PLA electrospun mats was investigated to suppress cervical carcinoma in vivo. A significant reduction in tumor volume was reported, and the recovery of half of the tumor-bearing mice within 3 weeks was achieved. Figure 3 shows the suppression results on tumor growth in this study. The significant reduction of tumor volume and weight was assumed to be caused by synergistic necrosis of the tumor cells, which was associated with the multiple adenosine- $5^{\prime}$-triphosphate depletion promoted by high dosage of dichloroacetate. ${ }^{99}$

Using the technique of multilayer nanofiber production, the researchers coated paclitaxel-loaded CS nanofibers with hyaluronic acid, taking advantage of the interaction between positively charged CS nanofibers and negatively charged hyaluronic acid. Cell-culture results confirmed the ability of released paclitaxel to inhibit the attachment and proliferation of DU145 prostate cancer cells even in the presence of small quantities of the drug. ${ }^{6}$ However, some drawbacks of this method include burst release of paclitaxel within the first 48 hours. Compared to synthetic anticancer drugs, natural therapeutic agents with known anticancer properties and reduced side effects are of particularly great interest for cancer therapy. ${ }^{95,100}$ In this respect, green tea polyphenol (GTP)-loaded nanofibers were fabricated by Shao et al to preserve the chemical structure of bioactive substances before releasing into the medium. For this purpose, GTP was first noncovalently adsorbed on the surface of MWCNTs, followed by incorporation into PCL nanofibers. Using this technique, the burst release of GTP was considerably reduced during the first 2 days and showed low cytotoxicity to normal osteoblast cells, but high antiproliferative effect to tumor cells (human hepatocellular carcinoma cells, Hep G2). ${ }^{100}$ Inorganic compounds such as cisplatin are another class of materials with confirmed antitumor activities, but these have the problem of short half-life in the biological environment. As a method
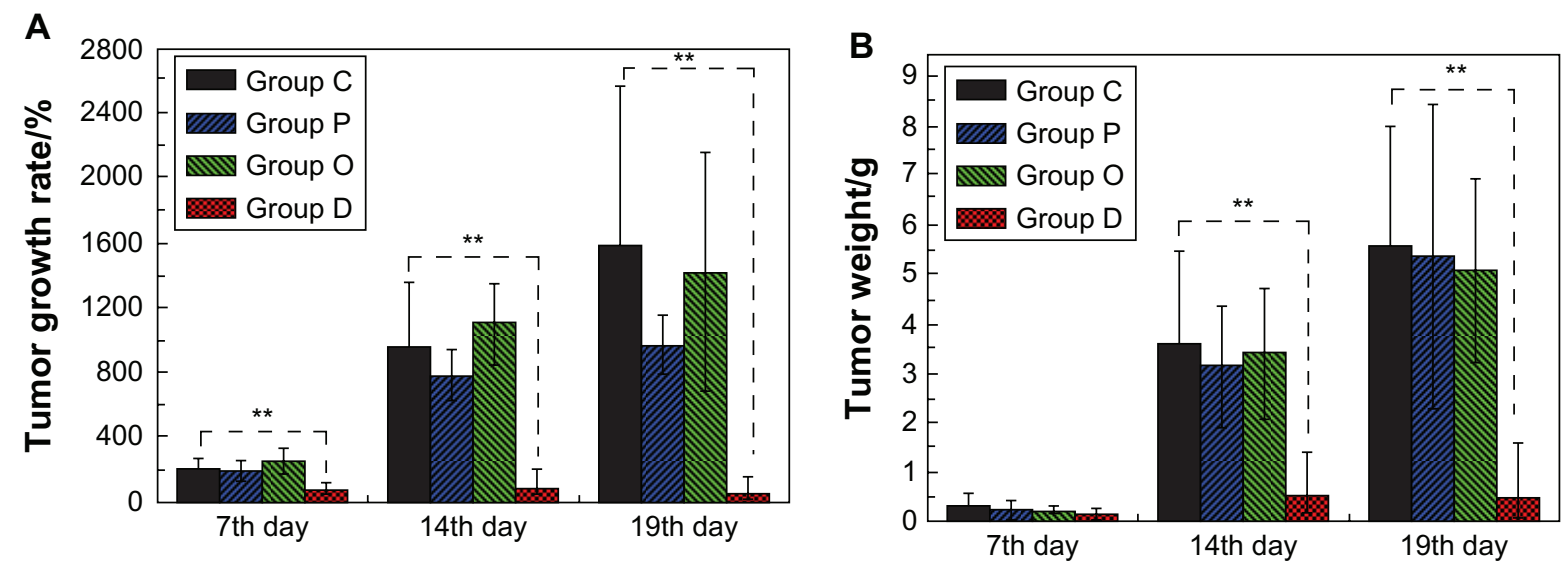

C

D
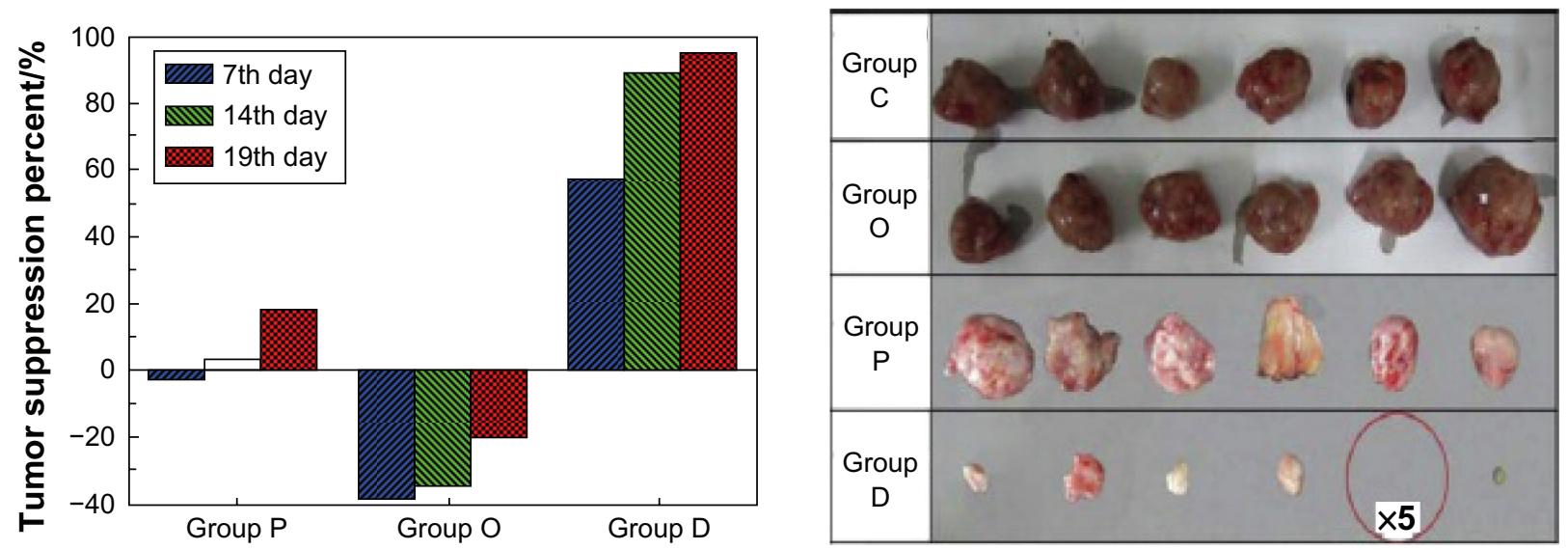

Figure 3 (A-D) Suppression results on tumor growth studied by Liu et al. ${ }^{99}$ Changes in tumor volume (A), tumor weight (B) and tumor suppression degree (C), and photos of tumors on the 19th day (D). No solid tumors were seen in five of the ten tumor-bearing mice in group $D$.

Notes: Control group (group C), blank PLA mat group (group P), orally administered group (group O) and DCA-loaded mat group (group D); **Highly significant differences between groups at a level of $P \leq 0.01$.

Reprinted from Biomaterials. Liu D, Liu S, Jing X, Li X, Li W, Huang Y. Necrosis of cervical carcinoma by dichloroacetate released from electrospun polylactide mats. 33(I7):4362-4369. Copyright (20I2) with permission from Elsevier. ${ }^{99}$ 
for improving the efficiency of inorganic anticancer drugs, titanocene dichloride was incorporated in PLLA nanofibers and showed inhibitory activity against lung tumor cells. ${ }^{102}$ For extremely hydrophobic antitumor agents, poor solubility and instability makes it difficult to have sustained release of active drug molecules with suitable concentration within a sufficient period of time. To solve these problems, hydroxycamptothecin (HCPT), an insoluble and unstable anticancer drug, was loaded in poly(D,L-lactic acid)-PEG electrospun nanofibers using 2-hydroxypropyl- $\beta$-cyclodextrin (HPCD) as the solubilizer. HCPT-loaded electrospun fibers caused much higher inhibitory activity against human mammary gland MCF-7 cancer cells compared to free drug during the first 72 hours of incubation. However, a biphasic release (a significant initial burst release followed by very slow or negligible release during incubation time) was observed, ${ }^{13}$ and to overcome this problem, emulsion electrospinning of HCPT was investigated in the presence of HPCD to fabricate core-shell nanofibers. Compared with the blend electrospinning technique, constant release was achieved due to the formation of preferential HCPT/HPCD complexes. Interestingly, the core-shell HCPT-loaded fibers showed higher inhibitory activity ( $>20$ times) against human hepatocellular carcinoma cells (Hep G2) than free HCPT during 72 hours' incubation, which was the direct consequence of the preservation of the chemical structure of HCPT for over a month. ${ }^{10}$

\section{DNA and siRNA delivery}

DNA and small interfering RNA (siRNA) are biomacromolecules with the ability to interfere with cellular processes by enhancing or preventing specific cues, which can be helpful in regenerative medicine. Slow release of DNA from tissueengineered scaffolds can expedite cell transfection, secretion of encoded GFs, and other signaling bioactive molecules. ${ }^{67} \mathrm{In}$ general, gene-delivery systems can be divided into two main categories: viral and nonviral delivery systems. Generally, the type of cells and viruses, gene structure, and the utilized delivery technique determine the degree of success in viral gene delivery. Developing novel and more efficient carriers, particularly polymeric carriers, for viral vector delivery is necessary to benefit from in situ viral delivery of genes over application of pretransduced cells. ${ }^{66}$ In contrast to viral gene delivery, nonviral vectors, such as naked pDNA and its complex with cationic agents, do not have the risk of toxicity, and a wider range of DNAs with various sizes can be used, though this approach usually suffers from low transfection efficiency. ${ }^{103}$ In addition to transfection efficiency, cell viability is of great importance in designing delivery systems. Features such as high surface area, high porosity, interconnected pores beneficial for cell adhesion/proliferation and oxygen/nutrient transferral, ${ }^{3}$ and loose bonding between fibers favorable for cell migration and infiltration ${ }^{78}$ caused electrospun nanofibers to be used as scaffolds for delivery of nucleic acids, eg, DNA and siRNA. Different attempts have been made to produce DNA-functionalized nanofibrous scaffolds for gene delivery in tissue engineering. $3,59,65-67,76,78,104,105$ Among the different techniques, the blending of DNA with an electrospinning solution did not cause satisfactory results, due to improper encapsulation and transfection efficiency. ${ }^{65}$ In order to overcome the low transfection efficiency of naked DNA-loaded nanofibers, incorporation of DNA-loaded particles into nanofibers, ${ }^{105}$ core-shell nanofibers, ${ }^{66,67}$ and surface modification $^{3,59}$ was examined. Figure 4 shows examples of techniques employed for DNA incorporation into nanofibrous scaffolds and their outstanding outcomes.

siRNA is a class of bioactive macromolecules with proven ability to silence the expression of specific proteins, a useful phenomenon when the tissue-repair process is prohibited by the secretion of inhibitory factors, or in cancer therapy, where specific genes can enhance tumor growth. ${ }^{106} \mathrm{Cao}$ et al published the first report on encapsulating siRNA within PCL nanofibers. The bioactivity of incorporated glyceraldehyde-3phosphate dehydrogenase siRNA was preserved during electrospinning, leading to repression efficiency of $61 \%-81 \%$ (in the presence of transfection reagent TransIT-TKO ${ }^{\mathrm{TM}}$ [Mirus Bio LLC, Madison, WI, USA]), as well as higher cellular uptake and successful transfection of human embryonic kidney 293 cells. ${ }^{43}$ However, due to the hydrophobic properties and slow degradation of PCL nanofibers, siRNA release rate was significantly slow. In order to achieve faster siRNA release and also to enhance gene-silencing efficiency, siRNA and transfection reagent TKO complexes encapsulated into poly(caprolactone-co-ethyl ethylene phosphate) increased the siRNA release rate, and mouse fibroblast NIH 3 T3 cells seeded on the scaffolds showed significant gene silencing even in the absence of transfection reagent. ${ }^{47}$ Surface functionalization of nanofibers as well as incorporation of siRNA-loaded nanoparticles into the electrospun nanofibers were also examined to investigate the release mechanism and achieve matrix metalloproteinase-responsive release of siRNA, respectively. ${ }^{107,108}$

\section{Growth-factor delivery}

GFs are another group of biomacromolecules capable of regulating biological processes by transferring signals between cells and their ECM, regulating proliferation, migration, and differentiation of cells, thereby enhancing tissue regeneration. ${ }^{109}$ Thus, the integration of GFs into 


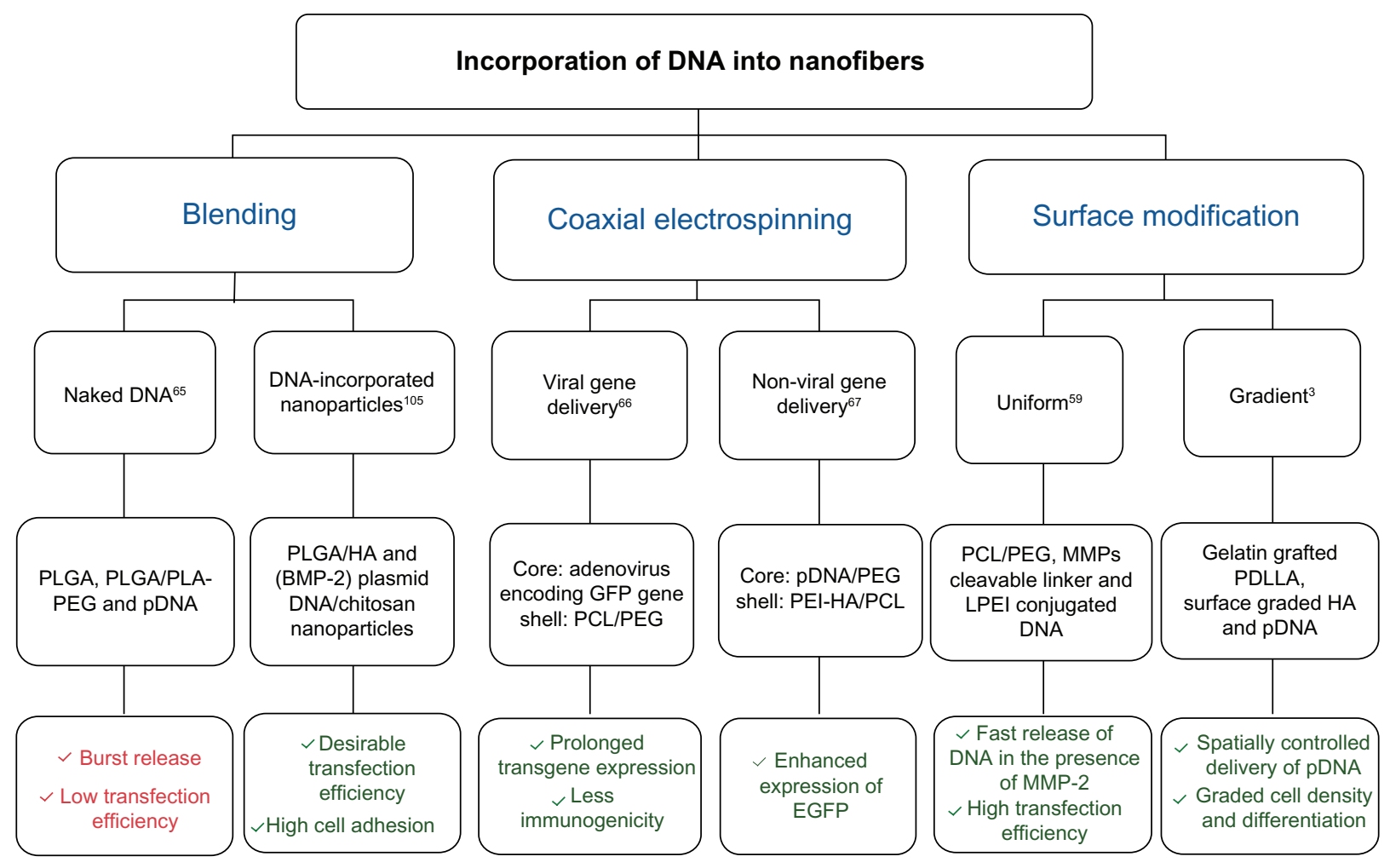

Figure 4 Representative studies on the fabrication of DNA-functionalized nanofibrous scaffolds via various techniques. Notes: Red color, unsatisfactory results; Green color, satisfactory results.

Abbreviations: HA, hydroxyapatite; BMP-2, bone morphogenetic protein-2; GFP, green fluorescent protein; PEI, polyethyleneimine; MMPs, matrix metalloproteinases; LPEI, linear polyethyleneimine; PDLLA, poly(D,L-lactic); pDNA, plasmid DNA; PLGA, poly(lactic-co-glycolic acid); PLA, poly(lactic acid); PEG, polyethylene glycol; PCL, poly( $\varepsilon$ caprolactone); EGFP, enhanced green fluorescent protein.

ECM-mimicking scaffolds could be beneficial, especially if the damaged tissues do not possess the required potential for regeneration. ${ }^{110}$ Although the controlled release of GFs is achievable, the instability of GFs hinders the successful development of GF-loaded tissue-engineering scaffolds. Various techniques, such as blending, ${ }^{91,111}$ specific or nonspecific surface modifications, , $7,60-63,105^{7}$ coaxial electrospinning, ${ }^{56}$ emulsion electrospinning, ${ }^{79,88}$ and combination of electrospinning with other conventional techniques, ${ }^{112,113}$ were applied for GF incorporation into nanofibrous scaffolds, yielding varied level of success. Burst release of EGF was obtained from silk nanofibers blended with EGF, due to the hydrophobic nature of EGF. ${ }^{91}$ However, a surface-modified nanofibrous scaffold was more appropriate for GF delivery, mainly due to its morphological and biochemical similarity to native tissues. ${ }^{57}$

Among the different substances with capability of conjugation with GFs, heparin remains the perfect choice, since it provides a more mimicked native ECM composition with the ability to efficiently interact with GF via negatively charged sulfate groups on its backbone. ${ }^{57,63}$ Moreover, polysaccharides that are found in proteoglycans (eg, aggrecan) of mammalian tissues are able to conjugate to GFs in a similar way to natural ECM. Mimicking the native tissues, attempts have been made to immobilize GF onto scaffolds using polysaccharides and/or heparin. ${ }^{57,60,63}$ Mottaghitalab et al assessed nerve GF (NGF) surface-conjugated CS/PVA scaffolds with respect to adhesion and proliferation of SKNMC (human neuroblastoma) and U373 (human glioblastomaastrocytoma) cell lines. However, using this technique, an initial release of localized NGF on the surface followed by diffusion-based release of NGF was observed. ${ }^{60}$ To solve the problem of GF burst release, electrospun CS fibers were surface-immobilized with heparin-containing polyelectrolyte complex nanoparticles (PCNs) presenting basic fibroblast GF (FGF-2), which were adsorbed to the nanofibers' surface. As a result, 30 days' zero-order release of FGF-2/PCN was achieved taking advantage of the preservative properties of heparin against proteolytic and chemical inactivation of GFs. bFGF/PCN complexes exhibited mitogenic activity to ovine bone marrow-derived mesenchymal stem cells, even after 27 days of incubation at $37^{\circ} \mathrm{C}$ in solution. ${ }^{57}$ However, the three-step procedure involves much complexity compared to other commonly used procedures. 
Moreover, preservation of bioactivity of GFs can ideally be obtained via coaxial electrospinning. Controlled delivery of vascular endothelial GF C (VEGFC), VEGFA, and plateletderived GF BB from nanofibers for stimulation of local lymphatic and vascular systems, respectively, was described by Liao and Leong. Sustained release of GFs from coreshell polyurethane nanofibers over a period of 10-14 days was obtained with bioactivity comparable to fresh GFs for treatment of hemophilia. In vivo results were in good agreement with described in vitro results in this study. ${ }^{56}$ Core-shell bFGF-loaded nanofibers were also prepared by emulsion electrospinning, and in vivo studies demonstrated higher wound recovery with complete reepithelialization without fiber fragmentation for 4 weeks. Figure 5 shows the structure of core-shell fabricated fibers as well as representative images of treated and untreated wounds of this study. ${ }^{79}$

Besides the common techniques, such as surface modification and coelectrospinning, researchers tried to develop new GF-delivery systems using polymeric nanofibers in combination with other existing forms of biomaterials. Han et al tried to combine hydrogels and electrospun nanofibers into a sandwich structure to provide a better control over NGF delivery as well as preserve its bioactivity. Combination of hydrophilic PEG-PCL diacrylate hydrogel containing NGF with PCL electrospun nanofibrous membrane notably decreased the burst release of GF, resulting in a longer release time scale. Moreover, the bioactivity of released NGF was confirmed by neurite extension of PC12 cells derived from
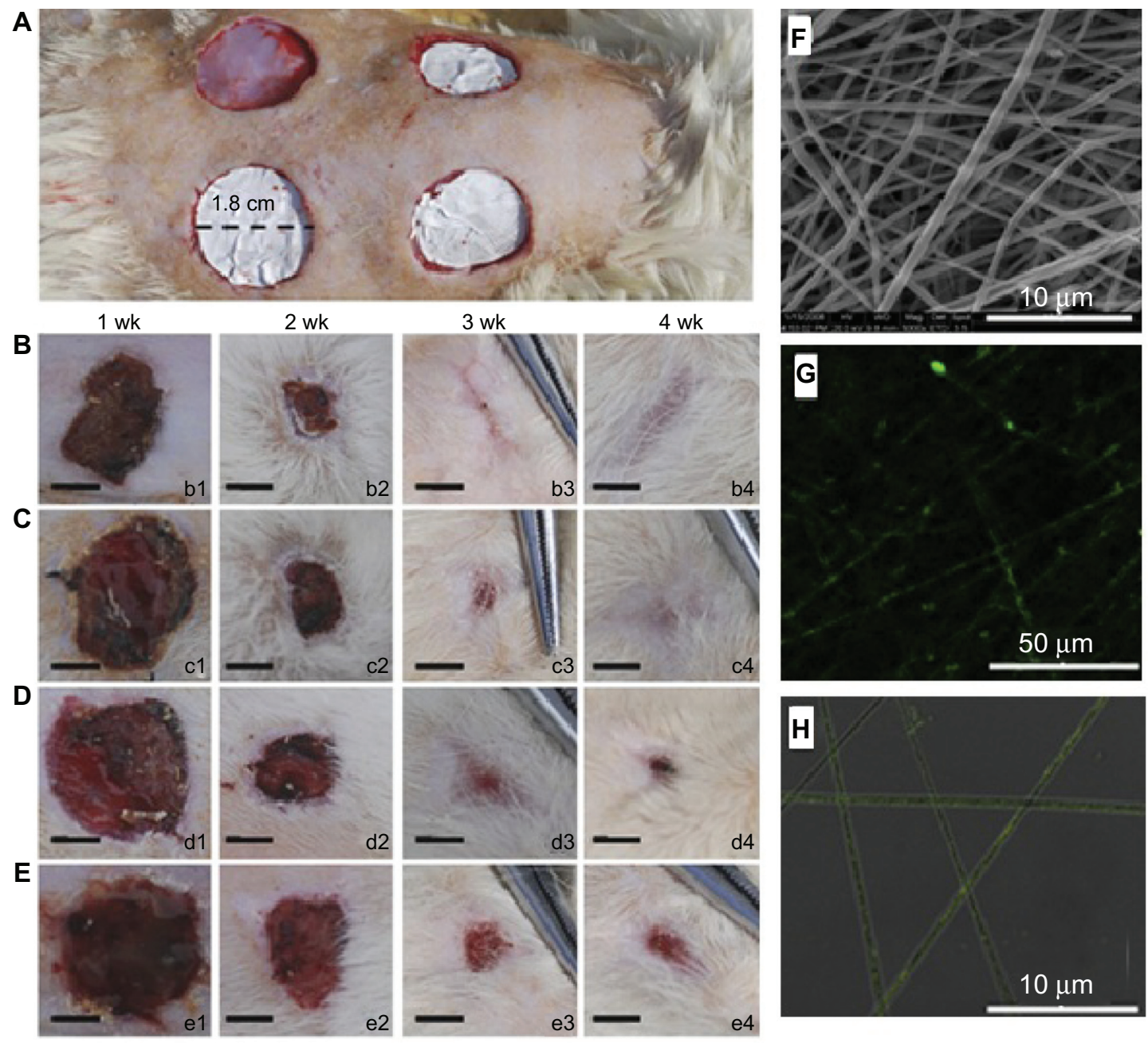

Figure 5 (A) Representative images of skin wounds covered with fibrous mats and the untreated wound surface. (B-E) The representative images of skin wounds after treatment with basic fibroblast growth factor (bFGF)/poly(D,L-lactic acid)-poly(ethylene glycol) (PELA) fibrous mats (b), and PELA mats with (c) or without free bFGF addition (d) for I, 2, 3, and 4 weeks, using untreated wound as control (e). Bars represent $10 \mathrm{~mm}$. (F) Scanning electron microscopy and (G) confocal laser scanning microscopy images of bFGF/PELA fibers; $(\mathbf{H})$ the images of the same fiber with and without excitation superimposed on one another.

Reprinted from Biomaterials. Yang Y, Xia T, Zhi W, et al. Promotion of skin regeneration in diabetic rats by electrospun core-sheath fibers loaded with basic fibroblast growth factor. 32(I8):4243-4254. Copyright (20II) with permission from Elsevier. ${ }^{79}$ 
pheochromocytoma of the rat adrenal medulla. ${ }^{113}$ However, the retention of a considerable portion of the originally loaded NGF within the hydrogel remained challenging.

\section{Most innovative applications}

Electrospun nanofibers containing antibiotics for periodontitis $^{45,114}$ or prevention of postsurgical adhesion/infections, ${ }^{8,115,116}$ analgesic and antipyretic, ${ }^{46,117}$ antiproliferative, ${ }^{118,119}$ anti-inflammatory, and anti-HIV120 are several examples of other nanofibrous drug-delivery systems that were developed for curing different target diseases. In the case of periodontal diseases, controlled local administration of antibiotics is considered effective, since the drug-delivery system can be placed directly in the periodontal pocket, achieving effective concentrations. In this way, the antibiotic agent would be available in the periodontal pocket in adequate concentrations for a sufficiently long period of time in spite of high sulcular fluid rates. An ideal implantable drug-delivery device should also be able to be easily inserted in the periodontal pocket and remain smooth and flexible for the required period of time in order to increase patient compliance. The degradability of the drug-delivery device can eliminate the risk of tissue damage that would be associated with the removal of nonbiodegradable devices after the treatment. ${ }^{45}$ Choosing proper materials, biodegradable nanofibers containing antibiotics can present an ideal drug-delivery system for periodontal treatment. For example, PCL and PLA were successfully applied as a carrier for metronidazole, resulting in sustained drug release over 19 days from the PCL matrix and release of $32 \%-48 \%$ of total drug content within 7 days from PLA carrier, respectively. ${ }^{45,114}$

$\mathrm{HIV}$ is another challenge for biomedical scientists, and a lot of attempts have been made to effectively prevent infection, though the complications in fighting the virus are known. ${ }^{121}$
Intravaginal delivery of microbicides, with the ability of elimination of microbes, including viruses, is a procedure for preventing virus transmission. Though gel-based systems are the most common formulations for vaginal microbicide delivery, there are some drawbacks, such as the high probability of the drug to be washed away with urine, resulting in decreased potency and causing practical inconveniences. ${ }^{122}$ Recently, the feasibility of using electrospun cellulose acetate phthalate (CAP) fibers containing the reverse-transcriptase inhibitor TMC 125 or tenofovir disoproxil fumarate (Viread) anti-HIV drugs was demonstrated by Huang and coworkers. Taking advantage of the $\mathrm{pH}$-dependent solubility of CAP in this formulation, the drug-release rate was modulated. That is, while CAP is stable in healthy vaginal fluid (acidic conditions), the presence of small amounts of human semen with $\mathrm{pH}$ 7.4-8.4 leads to immediate dissolution of nanofibers and subsequent release of incorporated drugs. Thus, the released antiviral drugs together with intrinsic antimicrobial properties of CAP were demonstrated to efficiently inhibit HIV transmission. Figure 6 shows a schematic illustration of the inhibitory action of CAP nanofibers containing the antiviral drugs. ${ }^{120}$

Besides the commonly known implantable nanofibrous drug-delivery systems, few studies ${ }^{84,123}$ have been published on the application of drug-loaded nanofibers as oral drug-delivery systems, which is the preferred route of drug administration from the aspects of convenience, patient compliance, and cost-effectiveness. Eudragit L 100-55 nanofibers containing diclofenac sodium, a nonsteroidal anti-inflammatory drug, were fabricated for oral drug-delivery purposes. PH-dependent drug release from Eudragit L 100-55 nanofibers was verified, with negligible release at $\mathrm{pH} 1.0$, but a sustained and complete release at $\mathrm{pH} 6.8$ was achieved. The release characteristics indi-

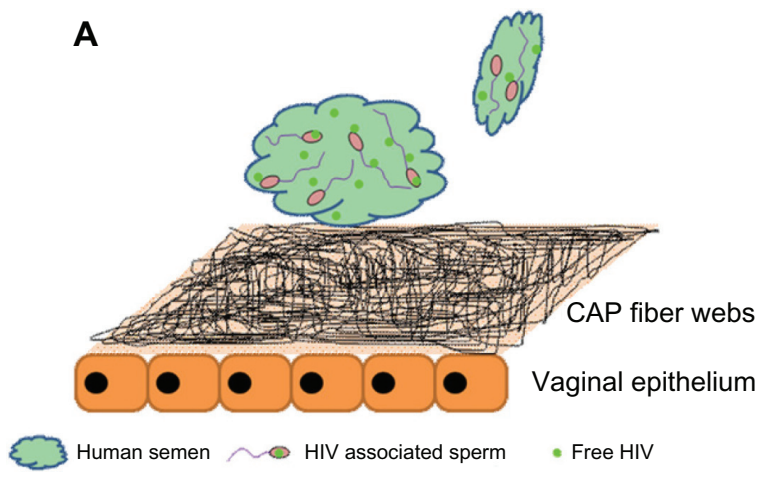

B

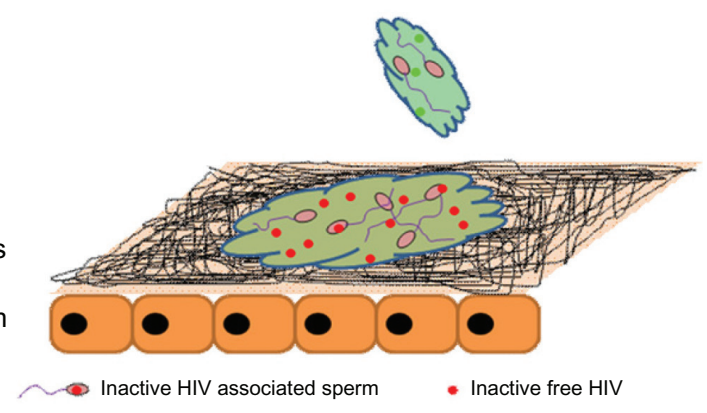

Figure 6 Schematic representation of vaginal epithelium covered by a web of electrospun cellulose acetate phthalate (CAP) fibers containing the antiviral drug (A) before contacting with human semen contaminated with HIV, and (B) after contacting with human semen contaminated with HIV.

Reprinted from Biomaterials. Huang C, Soenen SJ, van Gulck E, et al. Electrospun cellulose acetate phthalate fibers for semen induced anti-HIV vaginal drug delivery. 33(3):962-969. Copyright (2012) with permission from Elsevier. ${ }^{120}$ 
cate the ability of drug-loaded Eudragit L 100-55 nanofibers to be used as an oral colon-targeted drug-delivery system. ${ }^{123}$

\section{Electrosprayed particles as drug-delivery system}

In the past few years, micro/nanoparticles received growing interest for drug-delivery applications due to their release characteristics compared to previously developed delivery systems. Drug-loaded micro/nanoparticles can be used via most of the possible drug-administration routes, such as oral, injection, inhalation, and ophthalmic administration, and are greatly capable of facilitating both passive and active drug targeting. Compared to electrospun nanofibers, encapsulation of therapeutic agents into electrosprayed polymeric particles is a new approach in the development of drug-delivery vehicles. Cancer therapy has benefited a lot from polymeric particles for targeting the tumors via intravenous injection or local administration compared to other drugloaded carriers (eg, micelles, liposomes, etc). In addition to the typical advantages of particle-based anticancer drug-delivery systems, Ding et al proved that electrosprayed particles are more efficiently taken up by cells. This greater uptake was due to the surface charges of particles, which enhance particle adhesion on the surface of the cells, resulting in much higher probability of particle uptake. ${ }^{33}$

Postsurgical chemotherapy of glioma is a challenge in cancer therapy, due to limited permeability of injected drugs across the blood-brain barrier. Therefore, the application of drug-incorporated biodegradable polymeric devices as local implantable biomaterials holds great potential. ${ }^{124}$ Micro/nanoparticles can be safely and precisely inserted by stereotaxy into the target tissue of the brain, ${ }^{36}$ and also offer the possibility of transferring bioactive agents across the cell membrane. ${ }^{125}$ Microspheres are also able to carry the drug into remote areas in the formed cavity after surgical debulking by cerebrospinal fluid flow and edema. However, the retention of particles in the cavity for an adequate period of time along with its initial burst release remained problematic. ${ }^{40}$

EHDA was utilized for fabrication of paclitaxel-loaded PLGA microspheres that were subsequently entrapped in electrosprayed alginate hydrogel beads. The hydrogel matrixentrapped PLGA-paclitaxel microspheres showed sustained drug release over 60 days at near-zero-order kinetics with low initial burst release. Preserved cytotoxicity of paclitaxel in this formulation against $\mathrm{C} 6$ glioma was confirmed by cell-culture studies. As a result, electrosprayed paclitaxelloaded particles were more successful in subcutaneous tumor-volume reduction compared to Taxol and the control. ${ }^{40}$ In addition to glioma treatment, gliadin and gliadin-gelatin composite nanoparticles were synthesized for the controlled release of cyclophosphamide, an anticancer drug for breast cancer therapy. Changing the polymeric composition of nanoparticles made it possible to manipulate the cyclophosphamide release rate. By incorporation of gelatin into the gliadin matrix, the release rate was accelerated within 48 hours of drug release. Moreover, the apoptotic effect of cyclophosphamide-loaded 7\% gliadin nanoparticles against breast cancer cells cultured for 24 hours was confirmed by Western blot. ${ }^{22}$ Coaxial tricapillary electrospray was also used to produce multishell particles as drug-delivery systems for cancer therapy. Trilayered particles of PLGA-doxorubicin/ PDLLA(poly[D,L-lactic acid])-paclitaxel/PLGA showed low initial burst release, with the capability of controlling the release rate of individual drugs by altering polymer concentrations, flow rate, particle size, and shell thickness. With this technique, both paclitaxel and doxorubicin were released in stable, zero-order profiles. ${ }^{74}$

Besides polymeric particles, the production of solid-lipid particles via electrospraying for controlled delivery of insulin and tamoxifen was also attempted by Bussano et $\mathrm{al}^{50}$ and Trotta et al. ${ }^{126}$ EHDA can also be used in combination with other particle-producing methods such as complex coacervation, aiming to control the shape, size, and distribution of the produced particles. Combining electrospray-complex coacervation showed significant improvements in preparing CS-alginate particles. Alginate solution containing bovine serum albumin (BSA) was electrosprayed into the CS solution, and it produced monodisperse and spherical CSalginate particles. It was concluded that the particle size and BSA release rate were not dependent on the molecular weight of CS, while applied voltage influenced the particle size, shell thickness, and BSA-release rate. ${ }^{39}$

Even though electrospraying is a known process for particle fabrication, it was also introduced as an innovative method for film production instead of the common solutioncasting method. Electrosprayed and deposited films can be applied for local tumor chemotherapy or single-/multilayered coating of stents with drug-encapsulated polymers. ${ }^{127}$ Studies showed that paclitaxel molecules incorporated in electrosprayed PLGA film were in an amorphous state. Sustained release of paclitaxel from the films was achieved over 85 days, compared to the typical triphasic release profile of drug-loaded PLGA films. Phase-contrast microscopy and 3-(4,5-dimethylthiazol-2-yl)-2,5-diphenyltetrazolium bromide assay suggested sustained bioactivity of paclitaxel 
and slight reduction in the number of C6 glioma cells as a result of increased paclitaxel-loading concentration. ${ }^{127}$ Along with active particulate drug-delivery systems, smart multifunctional micro/nanoparticles based on polymeric or inorganic materials are receiving much interest due to their ability to target/image a specific tissue, followed by stimulated drug release via an external cue, such as ultrasound, $\mathrm{pH}$, temperature, or magnetic fields. These smart delivery systems enable controlled release of drugs as a response to changes in chemical or physical conditions. ${ }^{71}$ With the capability of electrospraying in producing inorganic particles, multifunctional paclitaxel-loaded core-shell particles with ultrasoundtriggered release properties were fabricated in a single step. Initial burst release together with paclitaxel release rate was successfully controlled by ultrasound stimulated cracking of the $\mathrm{TiO}_{2}$ shell. Moreover, the addition of $\mathrm{Fe}_{3} \mathrm{O}_{4}$ and graphene quantum dots into the core solution desirably performed magnetic targeting and fluorescence imaging, respectively. ${ }^{71}$ Figure 7 gives examples of the drug-release profiles from particles produced via various electrospraying techniques. $^{37,38,71,128}$

Electrosprayed polymeric particles are also beneficial for formulation of poorly water-soluble drugs for oral administration in order to shield the drug from biological processes, like undergoing high gut wall and first-pass metabolism. Coaxial EHDA was utilized for fabrication
A

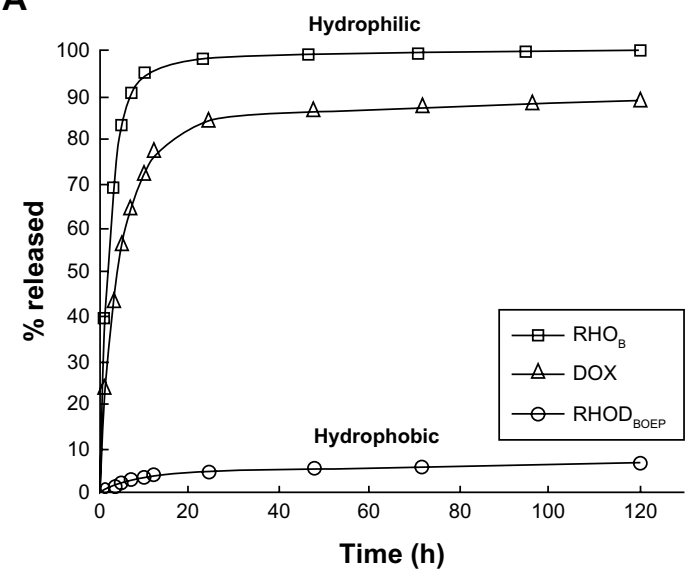

C

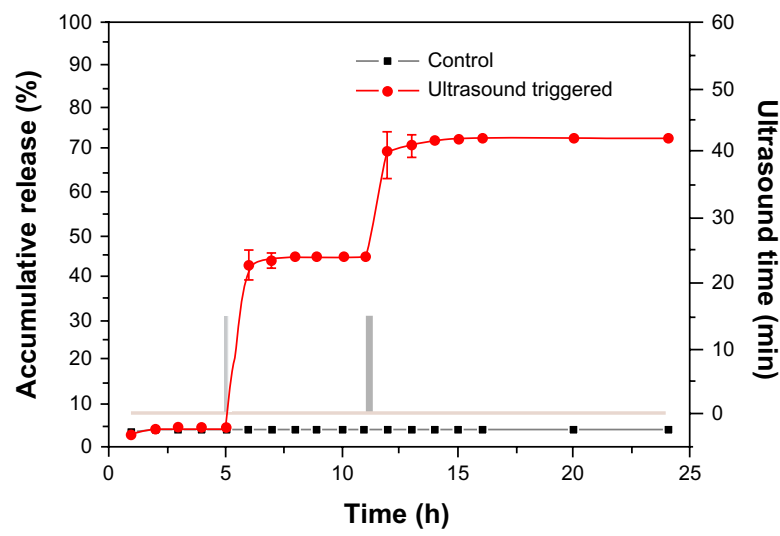

B

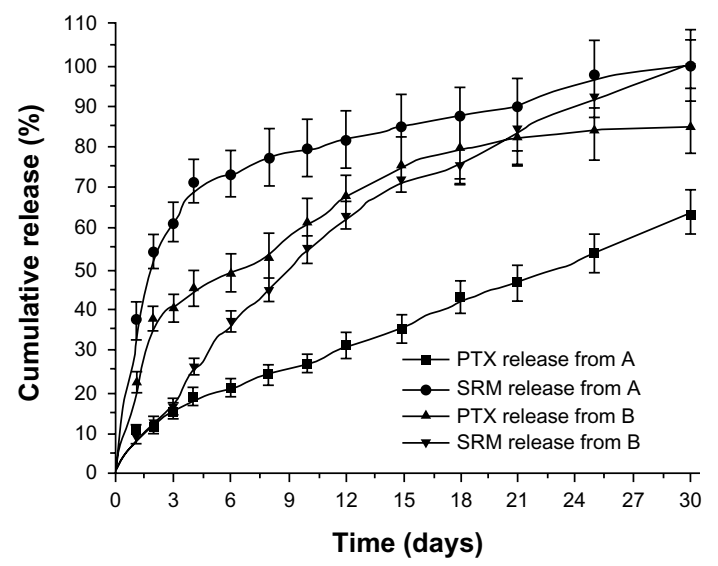

D

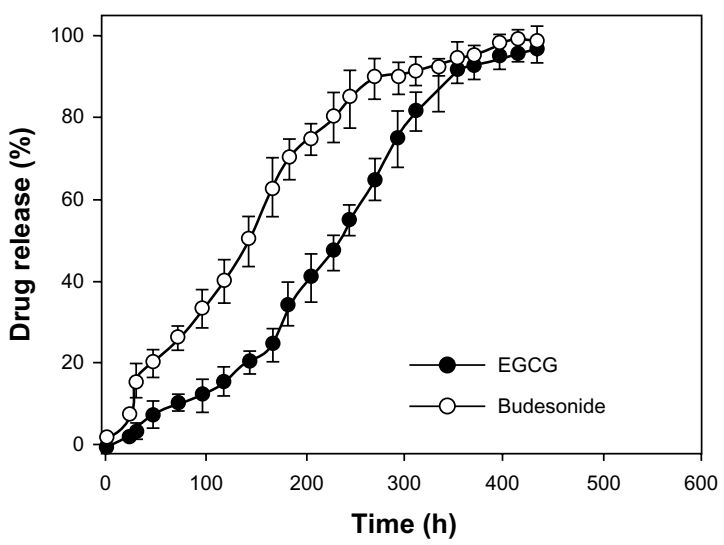

Figure 7 Drug-release profiles from (A) polyvinyl alcohol-coated PLGA particles, (B) PLLA/PLGA active coaxial dual-capillary electrosprayed particles (sample A: paclitaxel [PTX] encapsulated in the core and suramin in the shell and sample B vice versa), (C) smart $\mathrm{TiO}_{2}$ coaxial dual-capillary electrosprayed particles, (D) PLGA coaxial tricapillary electrosprayed particles (epigallocatechin gallate [EGCG] incorporated in core, budesonide in middle and PLGA in outer shell).

(A) Reprinted from J Control Release. Almería B, Fahmy TM, Gomez A. A multiplexed electrospray process for single-step synthesis of stabilized polymer particles for drug delivery. 154(2):203-210. Copyright (20II) with permission from Elsevier. ${ }^{38}$ (B) Reprinted from Biomaterials. Nie H, Fu Y, Wang CH. Paclitaxel and suramin-loaded core/ shell microspheres in the treatment of brain tumors. 3I(33):8732-8740. Copyright (2010) with permission from Elsevier. ${ }^{128}$ (C) Reprinted from Langmuir. Jing Y, Zhu Y, Yang X, Shen J, Li C. Ultrasound-triggered smart drug release from multifunctional core-shell capsules one-step fabricated by coaxial electrospray method. 27(3): I I 75-I I 80. Copyright (201 I) with permission from American Chemical Society. ${ }^{71}$ (D) Reprinted from Colloids Surf B Biointerfaces. Lee YH, Bai MY, Chen DR. Multidrug encapsulation by coaxial tri-capillary electrospray. 82(I): 104-II0. Copyright (201I) with permission from Elsevier. ${ }^{37}$

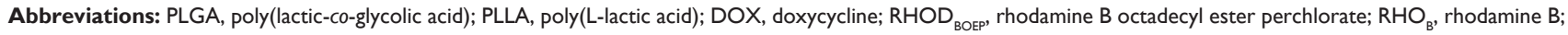
SRM, suramin; h, hours. 
of core-shell PLGA particles containing estradiol, the prescribed therapeutic agent for menopausal symptoms and prevention of postmenopausal osteoporosis. With this technique, an encapsulation efficiency of about $70 \%$ was obtained with sustained drug release over a period of 20 days. $^{49}$ Table 1 shows a list of various electrosprayed particles employed as drug-delivery systems. Considering the advantages of electrospraying over conventional techniques for production of drug-loaded particles, there is still hope for further development of electrosprayed particles for other therapeutic applications.

\section{Future directions}

EHD techniques show great potential for controlled drugdelivery applications, and by careful selection of materials and processing conditions, encapsulation of suitable therapeutic agents is possible. Though the accomplished successes in this field are considerable, further investigations are

Table I Electrosprayed particles employed as drug-delivery systems

\begin{tabular}{|c|c|c|c|c|c|c|}
\hline Polymer & Drug & Technique & $\begin{array}{l}\text { Particle } \\
\text { size }(\mu \mathrm{m})\end{array}$ & EE (\%) & Application & Reference \\
\hline Gliadin, gliadin/gelatin & Cyclophosphamide & B & $0.2-0.4$ & $53-72$ & Cancer therapy & 22 \\
\hline PLGA & Rifampicin & $\mathrm{B}$ & $\sim 6-8$ & - & Tuberculosis therapy & 25 \\
\hline PLLA & $\begin{array}{l}\text { Beclomethasone } \\
\text { dipropionate, } \\
\text { salbutamol sulfate }\end{array}$ & B & $0.3-0.6$ & $54-56$ & Asthma, rhinitis & 31 \\
\hline PCL & Paclitaxel & B & $6-12$ & 77-98 & Cancer therapy & 33 \\
\hline PLA & Paclitaxel & B & - & - & Cancer therapy & 34 \\
\hline PLGA & Paclitaxel & B & $\sim 0.3-5$ & - & Cancer therapy & 35 \\
\hline PLGA & Paclitaxel & B & $14.2-15.2$ & $78-82$ & Cancer therapy & 36 \\
\hline PCL & Paclitaxel & B & $\sim 11.4$ & $\sim 81$ & & \\
\hline $\begin{array}{l}\text { Palmitic acid } \\
\text { (in presence of EC or } \mathrm{PL} \text { ) }\end{array}$ & Insulin & B & $0.9-1.2$ & $65-90$ & Diabetes & 50 \\
\hline PLGA & Paclitaxel & B & 15 & - & Cancer therapy & 124 \\
\hline $\begin{array}{l}\text { Stearic acid } \\
\text { (in presence of EC) }\end{array}$ & Tamoxifen & B & $\sim 0.9$ & $\sim 70$ & Breast cancer & 126 \\
\hline PLGA & Paclitaxel & B & - & $63-93$ & Ovarian tumors & 127 \\
\hline Elastin-like polypeptides & Doxorubicin & B & $0.1-0.6$ & - & Cancer therapy & 129 \\
\hline Chitosan & Doxorubicin & B-PESM & $\sim 0.3-0.6$ & $63-68$ & Cancer therapy & 30 \\
\hline PLGA & $\begin{array}{l}\text { Doxorubicin } \\
\text { Rhodamine B } \\
\text { Rhodamine B } \\
\text { octadecyl ester } \\
\text { perchlorate }\end{array}$ & B-PESM & $\begin{array}{l}0.6-I .3 \\
0.6-I .1 \\
I . I-I .2\end{array}$ & $\begin{array}{l}94-100 \\
94-100 \\
100\end{array}$ & Cancer therapy & 38 \\
\hline Alginate & Paclitaxel & B-PESM & $1610-1680$ & $97-98$ & Cancer therapy & 40 \\
\hline Chitosan/alginate & Camptothecin & B-PESM & $\sim 3.1$ & $\sim 74$ & Cancer therapy & 64 \\
\hline TM-HT-chitosan/alginate & Camptothecin & B-PESM & $\sim 3.4$ & $\sim 70$ & & \\
\hline Eudragit L-100* & Griseofulvin & $B$ and CODC & $\begin{array}{l}\sim 1.9 \\
1.7-2.7\end{array}$ & - & Antifungal & 51 \\
\hline PLGA & $\begin{array}{l}\text { Budesonide, } \\
\text { epigallocatechin gallate }\end{array}$ & CODC & $0.16-1.2$ & $\begin{array}{l}90-95 \\
88-92\end{array}$ & $\begin{array}{l}\text { Asthma/rhinitis } \\
\text { Cancer therapy }\end{array}$ & 27 \\
\hline PLGA & Estradiol & CODC & $0.2-2.5$ & $65-75$ & Menopausal symptoms & 49 \\
\hline Tetrabutyl titanium & Paclitaxel & CODC & $0.6-6$ & $\sim 95$ & Cancer therapy & 71 \\
\hline $\begin{array}{l}\text { PLGA } \\
\text { PLLA }\end{array}$ & Paclitaxel, suramin & CODC & $10-20$ & - & Solid tumor & 128 \\
\hline PLGA & $\begin{array}{l}\text { Budesonide, } \\
\text { epigallocatechin gallate }\end{array}$ & COTC & $2.8-3.3$ & $\begin{array}{l}87-92 \\
90-93\end{array}$ & $\begin{array}{l}\text { Asthma/rhinitis } \\
\text { Cancer therapy }\end{array}$ & 37 \\
\hline $\begin{array}{l}\text { PLGA } \\
\text { PLA }\end{array}$ & Paclitaxel, doxorubicin & COTC & $3.5-6.5$ & $\begin{array}{l}83-89 \\
85-90\end{array}$ & Cancer therapy & 74 \\
\hline
\end{tabular}

Note: *Poly(methacrylic acid-co-methyl methacrylate).

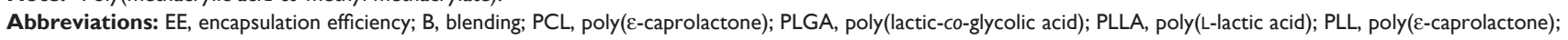
PLA, poly(D,L-lactic acid); EC, ethylcellulose; PL, Pluronic FI27; PESM, postelectrospray surface modification; TM-HT, N,N,N,-trimethylchitosan-homocysteine thiolactone; CODC, coaxial dual capillary; COTC, coaxial tricapillary. 
required to characterize precisely, previously produced drugdelivery systems, or to develop new materials or methods for improving the current products. This is especially required for incorporation of DNA, RNA, or GFs into the nanofibrous scaffolds for delivery of these biomacromolecules in a way very similar to the natural biological context. For this purpose, both the protein-release profile and bioactivity of the released protein need to be optimized. Also, the use of polymer blends or surface modification of nanofibers aiming to enhance the diffusion of medium to large drug molecules would bring additional advantages for TDDS. To date, most of the studies on drug release and cell nanofibrous membranes or cell-particle interactions have been performed in vitro, and in vivo studies are essentially needed to confirm the capability of the drug-loaded devices for clinical applications. However, while doing so, the drug-delivery system should also be preserved during preapplication procedures such as sterilization, and the probable impacts of biological events such as fibrosis or other immunological responses need evaluation. Moreover, with the proven feasibility of both electrospun nanofibers and electrosprayed particles for oral drug delivery, it is expected that these structures might gain great attention for oral drug delivery in the future. Further investigation on employing more suitable carriers for better preservation of therapeutic agents while traveling through the gastrointestinal tract is urgently required.

Coaxial techniques will be mainly useful for multidrugdelivery systems, whereby they provide the possibility of control over individual drug-release behavior, designing the surface properties without impairing the drug-incorporated core, and better preservation of bioactivity of unstable biomolecules. From the industrialization point of view, although the production rate of EHD methods in the single-jet mode is low, they are widely capable of scaling up using multiplex fluid jets. Currently, industrialized EHD setups are available, and there is also a great possibility of designing specific drug-delivery devices for special applications, taking advantage of the miniature model and versatile nature of EHD processes. Besides all positive functions of EHD techniques, there are still a few challenges associated with these methods that limit the complete success of this procedure. The lack of suitable EHD properties hinders the application of some useful materials, such as lipids, natural polymers, and ceramics, causing the need for additives such as polymers and organic or inorganic salts, which might not be biocompatible. In addition, reproducibility of the EHD processes remains a huge challenge due to the miniaturized nature of the process, which makes it almost impossible to control precisely the parameters on an industrial scale. Even with all these limitations, it is expected that functionalized nanofibers for delivery of biochemical cues into the body will become more competent in the near future. The systematic dealing with the existing challenges and difficulties involved in EHD processes makes it an active and flourishing field of research with a rapidly growing body of literature. ${ }^{129}$

\section{Acknowledgments}

This research was supported by NRF-Technion grant R-398001-065-592 and the Nanoscience and Nanotechnology Initiative, Faculty of Engineering, National University of Singapore, Singapore.

\section{Disclosure}

The authors report no conflicts of interest in this work.

\section{References}

1. Jain KK. Drug Delivery Systems, 1st ed. Hatfield, UK: Humana Press; 2008.

2. Suwantong O, Ruktanonchai U, Supaphol P. Electrospun cellulose acetate fiber mats containing asiaticoside or Centella asiatica crude extract and the release characteristics of asiaticoside. Polymer. 2008;49(19): 4239-4247.

3. Zou B, Liu Y, Luo X, Chen F, Guo X, Li X. Electrospun fibrous scaffolds with continuous gradations in mineral contents and biological cues for manipulating cellular behaviors. Acta Biomater. 2012;8(4): 1576-1585.

4. Yoo HS, Kim TG, Park TG. Surface-functionalized electrospun nanofibers for tissue engineering and drug delivery. Adv Drug Deliv Rev. 2009;61(12):1033-1042.

5. Cui W, Li X, Zhou S, Weng J. Investigation on process parameters of electrospinning system through orthogonal experimental design. J Appl Polym Sci. 2007;103(5):3105-3112.

6. Ma G, Liu Y, Peng C, Fang D, He B, Nie J. Paclitaxel loaded electrospun porous nanofibers as mat potential application for chemotherapy against prostate cancer. Carbohydr Polym. 2011;86(2):505-512.

7. Vargas EA, do Vale Baracho NC, de Brito J, de Queiroz AA. Hyperbranched polyglycerol electrospun nanofibers for wound dressing applications. Acta Biomater. 2010;6(3):1069-1078.

8. Zong X, Li S, Chen E, et al. Prevention of postsurgery-induced abdominal adhesions by electrospun bioabsorbable nanofibrous poly(lactide-coglycolide)-based membranes. Ann Surg. 2004;240(5):910-915.

9. Toshkova R, Manolova N, Gardeva E, et al. Antitumor activity of quaternized chitosan-based electrospun implants against Graffi myeloid tumor. Int J Pharm. 2010;400(1-2):221-233.

10. Luo X, Xie C, Wang H, Liu C, Yan S, Li X. Antitumor activities of emulsion electrospun fibers with core loading of hydroxycamptothecin via intratumoral implantation. Int J Pharm. 2012;425(1-2):19-28.

11. Zeng J, Yang L, Liang Q, et al. Influence of the drug compatibility with polymer solution on the release kinetics of electrospun fiber formulation. J Control Release. 2005;105(1-2):43-51.

12. Taepaiboon P, Rungsardthong U, Supaphol P. Drug-loaded electrospun mats of poly(vinyl alcohol) fibres and their release characteristics of four model drugs. Nanotechnology. 2006;17(9):2317-2329.

13. Xie C, Li X, Luo X, et al. Release modulation and cytotoxicity of hydroxycamptothecin-loaded electrospun fibers with 2-hydroxypropylbeta-cyclodextrin inoculations. Int J Pharm. 2010;391(1-2): 55-64. 
14. Rosca ID, Watari F, Uo M. Microparticle formation and its mechanism in single and double emulsion solvent evaporation. J Control Release. 2004;99(2):271-280.

15. Jain RA. The manufacturing techniques of various drug loaded biodegradable poly(lactide-co-glycolide) (PLGA) devices. Biomaterials. 2000;21(23):2475-2490.

16. Freytag T, Dashevsky A, Tillman L, Hardee GE, Bodmeier R. Improvement of the encapsulation efficiency of oligonucleotidecontaining biodegradable microspheres. J Control Release. 2000;69(1): 197-207.

17. Zambaux MF, Bonneaux F, Gref R, Dellacherie E, Vigneron C. Preparation and characterization of protein C-loaded PLA nanoparticles. J Control Release. 1999;60(2-3):179-188.

18. Freitas S, Merkle HP, Gander B. Microencapsulation by solvent extraction/evaporation: reviewing the state of the art of microsphere preparation process technology. J Control Release. 2005;102(2):313-332.

19. Mu L, Feng SS. Fabrication, characterization and in vitro release of paclitaxel (Taxol) loaded poly (lactic-co-glycolic acid) microspheres prepared by spray drying technique with lipid/cholesterol emulsifiers. J Control Release. 2001;76(3):239-254.

20. Omi S. Preparation of monodisperse microspheres using the Shirasu porous glass emulsification technique. Colloid Surfaces A. 1996; 109:97-107.

21. Singh M, O'Hagan D. The preparation and characterization of polymeric antigen delivery systems for oral administration. Adv Drug Deliv Rev. 1998;34(2-3):285-304.

22. Gulfam M, Kim JE, Lee JM, Ku B, Chung BH, Chung BG. Anticancer drug-loaded gliadin nanoparticles induce apoptosis in breast cancer cells. Langmuir. 2012;28(21):8216-8223.

23. Hans ML, Lowman AM. Biodegradable nanoparticles for drug delivery and targeting. Curr Opin Solid State Mater Sci. 2002;6(4):319-327.

24. Enlow EM, Luft JC, Napier ME, DeSimone JM. Potent engineered PLGA nanoparticles by virtue of exceptionally high chemotherapeutic loadings. Nano Lett. 2011;11(2):808-813.

25. Hong Y, Li Y, Yin Y, Li D, Zou G. Electrohydrodynamic atomization of quasi-monodisperse drug-loaded spherical/wrinkled microparticles. J Aerosol Sci. 2008;39(6):525-536.

26. Tamber H, Johansen P, Merkle HP, Gander B. Formulation aspects of biodegradable polymeric microspheres for antigen delivery. Adv Drug Deliv Rev. 2005;57(3):357-376.

27. Lee YH, Mei F, Bai MY, Zhao S, Chen DR. Release profile characteristics of biodegradable-polymer-coated drug particles fabricated by dual-capillary electrospray. J Control Release. 2010;145(1): 58-65.

28. Wang H, Liu Q, Yang Q, et al. Electrospun poly(methyl methacrylate) nanofibers and microparticles. J Mater Sci. 2009;45(4):1032-1038.

29. Xie J, Tan RS, Wang CH. Biodegradable microparticles and fiber fabrics for sustained delivery of cisplatin to treat $\mathrm{C} 6$ glioma in vitro. J Biomed Mater Res A. 2008;85(4):897-908

30. Songsurang K, Praphairaksit N, Siraleartmukul K, Muangsin N. Electrospray fabrication of doxorubicin-chitosan-tripolyphosphate nanoparticles for delivery of doxorubicin. Arch Pharm Res. 2011;34(4): 583-592.

31. Valo H, Peltonen L, Vehvilainen S, et al. Electrospray encapsulation of hydrophilic and hydrophobic drugs in poly(L-lactic acid) nanoparticles. Small. 2009;5(15):1791-1798.

32. Zauner W, Farrow NA, Haines AMR. In vitro uptake of polystyrene microspheres: effect of particle size, cell line and cell density. J Control Release. 2001;71(1):39-51.

33. Ding L, Lee T, Wang CH. Fabrication of monodispersed Taxol-loaded particles using electrohydrodynamic atomization. J Control Release. 2005;102(2):395-413.

34. Ciach T. Microencapsulation of drugs by electro-hydro-dynamic atomization. Int J Pharm. 2006;324(1):51-55.

35. Xie J, Lim LK, Phua Y, Hua J, Wang CH. Electrohydrodynamic atomization for biodegradable polymeric particle production. J Colloid Interface Sci. 2006;302(1):103-112.
36. Xie J, Marijnissen JC, Wang CH. Microparticles developed by electrohydrodynamic atomization for the local delivery of anticancer drug to treat C6 glioma in vitro. Biomaterials. 2006;27(17):3321-3332.

37. Lee YH, Bai MY, Chen DR. Multidrug encapsulation by coaxial tricapillary electrospray. Colloids SurfB Biointerfaces. 2011;82(1):104-110.

38. Almería B, Fahmy TM, Gomez A. A multiplexed electrospray process for single-step synthesis of stabilized polymer particles for drug delivery. $J$ Control Release. 2011;154(2):203-210.

39. Omidi J, Khorram M, Samimi A. Combined method of complex coacervation and electrospray for encapsulate preparation. J Appl Polym Sci. 2010;117(1):322-328.

40. Ranganath SH, Kee I, Krantz WB, Chow PK, Wang CH. Hydrogel matrix entrapping PLGA-paclitaxel microspheres: drug delivery with near zero-order release and implantability advantages for malignant brain tumour chemotherapy. Pharm Res. 2009;26(9):2101-2114.

41. Meng ZX, Xu XX, Zheng W, et al. Preparation and characterization of electrospun PLGA/gelatin nanofibers as a potential drug delivery system. Colloids Surf B Biointerfaces. 2011;84(1):97-102.

42. Kim K, Luu YK, Chang C, et al. Incorporation and controlled release of a hydrophilic antibiotic using poly(lactide-co-glycolide)-based electrospun nanofibrous scaffolds. J Control Release. 2004;98(1):47-56.

43. Cao H, Jiang $X$, Chai $C$, Chew SY. RNA interference by nanofiber-based siRNA delivery system. $J$ Control Release. 2010;144(2):203-212.

44. Jannesari M, Varshosaz J, Morshed M, Zamani M. Composite poly(vinyl alcohol)/poly(vinyl acetate) electrospun nanofibrous mats as a novel wound dressing matrix for controlled release of drugs. Int $J$ Nanomedicine. 2011;6:993-1003.

45. Zamani M, Morshed M, Varshosaz J, Jannesari M. Controlled release of metronidazole benzoate from poly epsilon-caprolactone electrospun nanofibers for periodontal diseases. Eur J Pharm Biopharm. 2010;75(2): 179-185.

46. Qi M, Li X, Yang Y, Zhou S. Electrospun fibers of acid-labile biodegradable polymers containing ortho ester groups for controlled release of paracetamol. Eur J Pharm Biopharm. 2008;70(2):445-452.

47. Rujitanaroj PO, Wang YC, Wang J, Chew SY. Nanofiber-mediated controlled release of siRNA complexes for long term gene-silencing applications. Biomaterials. 2011;32(25):5915-5923.

48. Kenawy ER, Abdel-Hay FI, El-Newehy MH, Wnek GE. Controlled release of ketoprofen from electrospun poly(vinyl alcohol) nanofibers. Mater Sci Eng A. 2007;459(1-2):390-396.

49. Enayati M, Ahmad Z, Stride E, Edirisinghe M. One-step electrohydrodynamic production of drug-loaded micro- and nanoparticles. J R Soc Interface. 2010;7(45):667-675.

50. Bussano R, Chirio D, Costa L, Turci F, Trotta M. Preparation and characterization of insulin-loaded lipid-based microspheres generated by electrospray. J Dispers Sci Technol. 2011;32(10):1524-1530.

51. Zhang S, Kawakami K, Yamamoto M, et al. Coaxial electrospray formulations for improving oral absorption of a poorly water-soluble drug. Mol Pharm. 2011;8(3):807-813.

52. Yao R, Zhang R, Luan J, Lin F. Alginate and alginate/gelatin microspheres for human adipose-derived stem cell encapsulation and differentiation. Biofabrication. 2012;4(2):025007.

53. Yao R, Zhang R, Wang X. Design and evaluation of a cell microencapsulating device for cell assembly technology. J Bioact Compat Pol. 2009;24 Suppl 1:48-62.

54. Xie J, Wang CH. Electrospray in the dripping mode for cell microencapsulation. J Colloid Interface Sci. 2007;312(2):247-255.

55. Xie J, Ng WJ, Lee LY, Wang CH. Encapsulation of protein drugs in biodegradable microparticles by co-axial electrospray. $J$ Colloid Interface Sci. 2008;317(2):469-476.

56. Liao IC, Leong KW. Efficacy of engineered FVIII-producing skeletal muscle enhanced by growth factor-releasing co-axial electrospun fibers. Biomaterials. 2011;32(6):1669-1677.

57. Volpato FZ, Almodovar J, Erickson K, Popat KC, Migliaresi C, Kipper MJ. Preservation of FGF-2 bioactivity using heparin-based nanoparticles, and their delivery from electrospun chitosan fibers. Acta Biomater. 2012;8(4):1551-1559. 
58. Im JS, Yun J, Lim YM, Kim HI, Lee YS. Fluorination of electrospun hydrogel fibers for a controlled release drug delivery system. Acta Biomater. 2010;6(1):102-109.

59. Kim HS, Yoo HS. MMPs-responsive release of DNA from electrospun nanofibrous matrix for local gene therapy: in vitro and in vivo evaluation. J Control Release. 2010;145(3):264-271.

60. Mottaghitalab F, Farokhi M, Mottaghitalab V, Ziabari M, Divsalar A, Shokrgozar MA. Enhancement of neural cell lines proliferation using nano-structured chitosan/poly(vinyl alcohol) scaffolds conjugated with nerve growth factor. Carbohydr Polym. 2011;86(2):526-535.

61. Choi JS, Leong KW, Yoo HS. In vivo wound healing of diabetic ulcers using electrospun nanofibers immobilized with human epidermal growth factor (EGF). Biomaterials. 2008;29(5):587-596.

62. Cho YI, Choi JS, Jeong SY, Yoo HS. Nerve growth factor (NGF)conjugated electrospun nanostructures with topographical cues for neuronal differentiation of mesenchymal stem cells. Acta Biomater. 2010;6(12):4725-4733.

63. Zou J, Yang Y, Liu Y, Chen F, Li X. Release kinetics and cellular profiles for bFGF-loaded electrospun fibers: effect of the conjugation density and molecular weight of heparin. Polymer. 2011;52(15): $3357-3367$.

64. Juntapram K, Praphairaksit N, Siraleartmukul K, Muangsin N. Electrosprayed polyelectrolyte complexes between mucoadhesive $\mathrm{N}, \mathrm{N}, \mathrm{N}$, -trimethylchitosan-homocysteine thiolactone and alginate/carrageenan for camptothecin delivery. Carbohydr Polym. 2012;90(4): 1469-1479.

65. Luu YK, Kim K, Hsiao BS, Chu B, Hadjiargyrou M. Development of a nanostructured DNA delivery scaffold via electrospinning of PLGA and PLA-PEG block copolymers. J Control Release. 2003;89(2):341-353.

66. Liao IC, Chen S, Liu JB, Leong KW. Sustained viral gene delivery through core-shell fibers. J Control Release. 2009;139(1):48-55.

67. Saraf A, Baggett LS, Raphael RM, Kasper FK, Mikos AG. Regulated non-viral gene delivery from coaxial electrospun fiber mesh scaffolds. $J$ Control Release. 2010;143(1):95-103.

68. He CL, Huang ZM, Han XJ, Liu L, Zhang HS, Chen LS. Coaxial electrospun poly(L-lactic acid) ultrafine fibers for sustained drug delivery. J Macromol Sci Phys. 2006;45(4):515-524.

69. Huang ZM, He CL, Yang A, et al. Encapsulating drugs in biodegradable ultrafine fibers through co-axial electrospinning. J Biomed Mater Res A. 2006;77(1):169-179.

70. He CL, Huang ZM, Han XJ. Fabrication of drug-loaded electrospun aligned fibrous threads for suture applications. J Biomed Mater Res A. 2009;89(1):80-95.

71. Jing Y, Zhu Y, Yang X, Shen J, Li C. Ultrasound-triggered smart drug release from multifunctional core-shell capsules one-step fabricated by coaxial electrospray method. Langmuir. 2011;27(3):1175-1180.

72. Briasoulis E, Karavasilis V, Tzamakou E, et al. Interaction pharmacokinetics of pegylated liposomal doxorubicin (Caelyx) on coadministration with paclitaxel or docetaxel. Cancer Chemother Pharmacol. 2004;53(5):452-457.

73. Gustafson DL, Merz AL, Long ME. Pharmacokinetics of combined doxorubicin and paclitaxel in mice. Cancer Lett. 2005;220(2): 161-169.

74. Kim W, Kim SS. Synthesis of biodegradable triple-layered capsules using a triaxial electrospray method. Polymer. 2011;52(15): 3325-3336.

75. Yang Y, Li X, Qi M, Zhou S, Weng J. Release pattern and structural integrity of lysozyme encapsulated in core-sheath structured poly(DLlactide) ultrafine fibers prepared by emulsion electrospinning. Eur $J$ Pharm Biopharm. 2008;69(1):106-116.

76. He S, Xia T, Wang H, Wei L, Luo X, Li X. Multiple release of polyplexes of plasmids VEGF and bFGF from electrospun fibrous scaffolds towards regeneration of mature blood vessels. Acta Biomater. 2012;8(7):2659-2669.

77. Xu X, Yang L, Wang X, et al. Ultrafine medicated fibers electrospun from W/O emulsions. J Control Release. 2005;108(1):33-42.
78. Yang Y, Li X, Cheng L, et al. Core-sheath structured fibers with pDNA polyplex loadings for the optimal release profile and transfection efficiency as potential tissue engineering scaffolds. Acta Biomater. 2011;7(6):2533-2543.

79. Yang Y, Xia T, Zhi W, et al. Promotion of skin regeneration in diabetic rats by electrospun core-sheath fibers loaded with basic fibroblast growth factor. Biomaterials. 2011;32(18):4243-4254.

80. Wang Y, Wang B, Qiao W, Yin T. A novel controlled release drug delivery system for multiple drugs based on electrospun nanofibers containing nanoparticles. J Pharm Sci. 2010;99(12):4805-4811.

81. Okuda T, Tominaga K, Kidoaki S. Time-programmed dual release formulation by multilayered drug-loaded nanofiber meshes. $J$ Control Release. 2010;143(2):258-264.

82. Xu J, Jiao Y, Shao X, Zhou C. Controlled dual release of hydrophobic and hydrophilic drugs from electrospun poly(1-lactic acid) fiber mats loaded with chitosan microspheres. Mater Lett. 2011;65(17-18):2800-2803.

83. Chunder A, Sarkar S, Yu Y, Zhai L. Fabrication of ultrathin polyelectrolyte fibers and their controlled release properties. Colloids Surf B Biointerfaces. 2007;58(2):172-179.

84. Im JS, Lee SK, Bai BC, Lee YS. Prediction and characterization of drug release in a multi-drug release system. $J$ Ind Eng Chem. 2012;18(1):325-330

85. Hong Y, Fujimoto K, Hashizume R, et al. Generating elastic, biodegradable polyurethane/poly(lactide-co-glycolide) fibrous sheets with controlled antibiotic release via two-stream electrospinning. Biomacromolecules. 2008;9(4):1200-1207.

86. Khil MS, Cha DI, Kim HY, Kim IS, Bhattarai N. Electrospun nanofibrous polyurethane membrane as wound dressing. J Biomed Mater Res B Appl Biomater. 2003;67(2):675-679.

87. Zhang Y, Lim CT, Ramakrishna S, Huang ZM. Recent development of polymer nanofibers for biomedical and biotechnological applications. J Mater Sci Mater Med. 2005;16(10):933-946.

88. Tian L, Prabhakaran MP, Ding X, Kai D, Ramakrishna S. Emulsion electrospun vascular endothelial growth factor encapsulated poly(1lactic acid-co- $\varepsilon$-caprolactone) nanofibers for sustained release in cardiac tissue engineering. J Mater Sci. 2011;47(7):3272-3281.

89. Boateng JS, Matthews KH, Stevens HN, Eccleston GM. Wound healing dressings and drug delivery systems: a review. J Pharm Sci. 2008;97(8): 2892-2923.

90. Said SS, Aloufy AK, El-Halfawy OM, Boraei NA, El-Khordagui LK. Antimicrobial PLGA ultrafine fibers: interaction with wound bacteria. Eur J Pharm Biopharm. 2011;79(1):108-118.

91. Schneider A, Wang XY, Kaplan DL, Garlick JA, Egles C. Biofunctionalized electrospun silk mats as a topical bioactive dressing for accelerated wound healing. Acta Biomater. 2009;5(7):2570-2578.

92. Yun J, Im JS, Lee YS, Kim HI. Electro-responsive transdermal drug delivery behavior of PVA/PAA/MWCNT nanofibers. Eur Polym $J$. 2011;47(10):1893-1902.

93. Prausnitz MR, Langer R. Transdermal drug delivery. Nat Biotechnol. 2008;26(11):1261-1268.

94. Prausnitz MR, Mitragotri S, Langer R. Current status and future potential of transdermal drug delivery. Nat Rev Drug Discov. 2004;3(2): $115-124$.

95. Suwantong O, Opanasopit P, Ruktanonchai U, Supaphol P. Electrospun cellulose acetate fiber mats containing curcumin and release characteristic of the herbal substance. Polymer. 2007;48(26):7546-7557.

96. Im JS, Bai B, Lee YS. The effect of carbon nanotubes on drug delivery in an electro-sensitive transdermal drug delivery system. Biomaterials. 2010;31(6):1414-1419.

97. Taepaiboon P, Rungsardthong U, Supaphol P. Vitamin-loaded electrospun cellulose acetate nanofiber mats as transdermal and dermal therapeutic agents of vitamin A acid and vitamin E. Eur J Pharm Biopharm. 2007;67(2):387-397.

98. Ngawhirunpat T, Opanasopit P, Rojanarata T, Akkaramongkolporn P, Ruktanonchai U, Supaphol P. Development of meloxicam-loaded electrospun polyvinyl alcohol mats as a transdermal therapeutic agent. Pharm Dev Technol. 2009;14(1):70-79. 
99. Liu D, Liu S, Jing X, Li X, Li W, Huang Y. Necrosis of cervical carcinoma by dichloroacetate released from electrospun polylactide mats. Biomaterials. 2012;33(17):4362-4369.

100. Shao S, Li L, Yang G, et al. Controlled green tea polyphenols release from electrospun PCL/MWCNTs composite nanofibers. Int J Pharm. 2011;421(2):310-320.

101. Pradilla G, Wang PP, Gabikian P, et al. Local intracerebral administration of paclitaxel with the paclimer delivery system: toxicity study in a canine model. J Neurooncol. 2006;76(2):131-138.

102. Chen P, Wu QS, Ding YP, Chu M, Huang ZM, Hu W. A controlled release system of titanocene dichloride by electrospun fiber and its antitumor activity in vitro. Eur J Pharm Biopharm. 2010;76(3): 413-420.

103. Yamamoto M, Tabata Y. Tissue engineering by modulated gene delivery. Adv Drug Deliv Rev. 2006;58(4):535-554.

104. Lee S, Kim JS, Chu HS, Kim GW, Won JI, Jang JH. Electrospun nanofibrous scaffolds for controlled release of adeno-associated viral vectors. Acta Biomater. 2011;7(11):3868-3876.

105. Nie H, Wang CH. Fabrication and characterization of PLGA/HAp composite scaffolds for delivery of BMP-2 plasmid DNA. J Control Release. 2007;120(1-2):111-121.

106. Meinel AJ, Germershaus O, Luhmann T, Merkle HP, Meinel L. Electrospun matrices for localized drug delivery: current technologies and selected biomedical applications. Eur J Pharm Biopharm. 2012; 81(1):1-13.

107. Kim HS, Yoo HS. Matrix metalloproteinase-inspired suicidal treatments of diabetic ulcers with siRNA-decorated nanofibrous meshes. Gene Ther. 2013;20(4):378-385.

108. Chen M, Gao S, Dong M, et al. Chitosan/siRNA nanoparticles encapsulated in PLGA nanofibers for siRNA delivery. ACS Nano. 2012;6(6):4835-4844.

109. Chen FM, Zhang M, Wu ZF. Toward delivery of multiple growth factors in tissue engineering. Biomaterials. 2010;31(24):6279-6308.

110. Tabata II. The importance of drug delivery systems in tissue engineering. Pharm Sci Technolo Today. 2000;3(3):80-89.

111. Zhang $\mathrm{X}$, Shi $\mathrm{Z}, \mathrm{Fu} \mathrm{W}$, et al. In vitro biocompatibility study of electrospun copolymer ethylene carbonate- $\varepsilon$-caprolactone and vascular endothelial growth factor blended nanofibrous scaffolds. Appl Surf Sci. 2012;258(7):2301-2306.

112. Kolambkar YM, Dupont KM, Boerckel JD, et al. An alginate-based hybrid system for growth factor delivery in the functional repair of large bone defects. Biomaterials. 2011;32(1):65-74.

113. Han N, Johnson J, Lannutti JJ, Winter JO. Hydrogel-electrospun fiber composite materials for hydrophilic protein release. J Control Release. 2012;158(1):165-170.

114. Reise M, Wyrwa R, Müller U, et al. Release of metronidazole from electrospun poly(L-lactide-co-D/L-lactide) fibers for local periodontitis treatment. Dent Mater. 2012;28(2):179-188.
115. Liu H, Leonas KK, Zhao Y. Antimicrobial properties and release profile of ampicillin from electrospun poly( $\varepsilon$-caprolactone) nanofiber yarns. $J$ Eng Fiber Fabr. 2010;5(4):10-19.

116. Bölgen N, Vargel I, Korkusuz P, Menceloğlu YZ, Pişkin E. In vivo performance of antibiotic embedded electrospun PCL membranes for prevention of abdominal adhesions. J Biomed Mater Res B Appl Biomater. 2007;81(2):530-543.

117. Peng H, Zhou S, Guo T, et al. In vitro degradation and release profiles for electrospun polymeric fibers containing paracetanol. Colloids Surf B Biomaterials. 2008;66(2):206-212.

118. Innocente F, Mandracchia D, Pektok E, et al. Paclitaxel-eluting biodegradable synthetic vascular prostheses: a step towards reduction of neointima formation? Circulation. 2009;120(11 Suppl 1): S37-S45.

119. Luong-Van E, Grondahl L, Chua KN, Leong KW, Nurcombe V, Cool SM. Controlled release of heparin from poly( $\varepsilon$-caprolactone) electrospun fibers. Biomaterials. 2006;27(9):2042-2050.

120. Huang C, Soenen SJ, van Gulck E, et al. Electrospun cellulose acetate phthalate fibers for semen induced anti-HIV vaginal drug delivery. Biomaterials. 2012;33(3):962-969.

121. Janssen RS, Holtgrave DR, Valdiserri RO, Shepherd M, Gayle HD, De Cock KM. The serostatus approach to fighting the HIV epidemic: prevention strategies for infected individuals. Am J Public Health. 2001;91(7):1019-1024.

122. Berger EA, Doms RW, Fenyö EM, et al. A new classification for HIV-1. Nature. 1998;391(6664):240.

123. Shen X, Yu D, Zhu L, Branford-White C, White K, Chatterton NP. Electrospun diclofenac sodium loaded Eudragit L 100-55 nanofibers for colon-targeted drug delivery. Int J Pharm. 2011;408(1-2):200-207.

124. Naraharisetti KP, Ong B, Xie WJ, Lee KYT, Wang CH, Sahinidis NV. In vivo performance of implantable biodegradable preparations delivering paclitaxel and etanidazole for the treatment of glioma. Biomaterials. 2007;28(5):886-894.

125. Arya N, Chakraborty S, Dube N, Katti DS. Electrospraying: a facile technique for synthesis of chitosan-based micro/nanospheres for drug delivery applications. J Biomed Mater Res B Appl Biomater. 2009; $88(1): 17-31$.

126. Trotta M, Cavalli R, Trotta C, Bussano R, Costa L. Electrospray technique for solid lipid-based particle production. Drug Dev Ind Pharm. 2010;36(4):431-438.

127. Xie J, Tan JC, Wang CH. Biodegradable films developed by electrospray deposition for sustained drug delivery. J Pharm Sci. 2008;97(8):3109-3122.

128. Nie H, Fu Y, Wang CH. Paclitaxel and suramin-loaded core/shell microspheres in the treatment of brain tumors. Biomaterials. 2010 31(33):8732-8740.

129. Wu Y, MacKay JA, McDaniel JR, Chilkoti A, Clark RL. Fabrication of elastin-like polypeptide nanoparticles for drug delivery by electrospraying. Biomacromolecules. 2009;10(1):19-24.
International Journal of Nanomedicine

\section{Publish your work in this journal}

The International Journal of Nanomedicine is an international, peerreviewed journal focusing on the application of nanotechnology in diagnostics, therapeutics, and drug delivery systems throughout the biomedical field. This journal is indexed on PubMed Central,

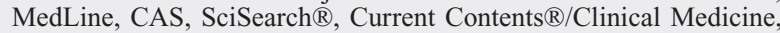

\section{Dovepress}

Journal Citation Reports/Science Edition, EMBase, Scopus and the Elsevier Bibliographic databases. The manuscript management system is completely online and includes a very quick and fair peer-review system, which is all easy to use. Visit http://www.dovepress.com/ testimonials.php to read real quotes from published authors. 